\title{
Effects of Memory Biases on Variability of Temperature Reconstructions ${ }^{\mathscr{O}}$
}

\author{
Lucie J. LÜCKe, GABriele C. Hegerl, AND ANDrew P. SCHURER \\ School of Geosciences, University of Edinburgh, Edinburgh, United Kingdom \\ ROB WILSON \\ School of Earth and Environmental Sciences, University of St Andrews, St Andrews, United Kingdom, and Tree-Ring \\ Laboratory, Lamont-Doherty Earth Observatory of Columbia University, Palisades, New York
}

(Manuscript received 6 March 2019, in final form 6 August 2019)

\begin{abstract}
Quantifying past climate variation and attributing its causes improves our understanding of the natural variability of the climate system. Tree-ring-based proxies have provided skillful and highly resolved reconstructions of temperature and hydroclimate of the last millennium. However, like all proxies, they are subject to uncertainties arising from varying data quality, coverage, and reconstruction methodology. Previous studies have suggested that biological-based memory processes could cause spectral biases in climate reconstructions. This study determines the effects of such biases on reconstructed temperature variability and the resultant implications for detection and attribution studies. We find that introducing persistent memory, reflecting the spectral properties of tree-ring data, can change the variability of pseudoproxy reconstructions compared to the surrogate climate and resolve certain model-proxy discrepancies. This is especially the case for proxies based on ring-width data. Such memory inflates the difference between the Medieval Climate Anomaly and the Little Ice Age and suppresses and extends the cooling in response to volcanic eruptions. When accounting for memory effects, climate model data can reproduce long-term cooling after volcanic eruptions, as seen in proxy reconstructions. Results of detection and attribution studies show that signals in reconstructions as well as residual unforced variability are consistent with those in climate models when the model fingerprints are adjusted to reflect autoregressive memory as found in tree rings.
\end{abstract}

\section{Introduction}

Long-term climate reconstructions from natural climate archives provide the basis for quantifying the full amount of natural climate variability and attributing variations to external forcings or chaotic internal fluctuations. While tree rings provide annually resolved and precisely dated climate signal (Stokes and Smiley 1968) and correlate well with observed temperature and precipitation records (Fritts 1976), they are subject to a wide range of uncertainties (e.g., Fritts 1976; Esper et al. 2004; Jones et al. 2009; Cook and Pederson 2010; Frank et al. 2010a). Here we focus on investigating

\footnotetext{
Supplemental information related to this paper is available at the Journals Online website: https://doi.org/10.1175/JCLI-D-190184.s1.
}

Corresponding author: Lucie Lücke, lucie.luecke@ed.ac.uk the impacts of spectral biases on temperature reconstructions from tree rings, specifically impacts on lowfrequency variability and response to volcanic forcing, and their implications for detection and attribution studies.

It is well known that physiological processes within a tree can affect the climate signal and induce a biologicalbased memory signal (Fritts 1976; Schulman 1956; Matalas 1962; Vaganov et al. 2010). Fritts (1976) suggests that the storage of sugar and hormones as well as the growth of leaves (needles), roots, and fruits could affect the persistence of the climate signal from one year to the next. Many studies have found that data based on ring width (RW) as a proxy for past temperature and precipitation contain more autocorrelation and long-term memory than data derived from maximum latewood density (MXD) (Esper et al. 2015; Franke et al. 2013; Zhang et al. 2016; Anchukaitis et al. 2012; Krakauer and Randerson 2003; Helama et al. 2009). It 
should, however, be noted that it is not clear why MXD data do not portray similar persistent properties as RW. It was observed that RW underestimates and temporally extends the response to volcanic eruptions compared to MXD (Frank et al. 2010a; D'Arrigo et al. 2013; Anchukaitis et al. 2012; Esper et al. 2015). Franke et al. (2013) found that RW temperature records are strongly red biased compared to observations, whereas the spectral characteristics of MXD data are in better agreement with observations, although they still seem biased regarding their ratio of low- to high-frequency variability. Furthermore, they found that these biases propagate into climate field reconstructions, which display significantly more memory than observations. Zhang et al. (2016) conducted pseudoproxy experiments in which they increased the memory in precipitation data from climate models for China. They observed that increased local-scale memory propagated into the pseudoproxy reconstruction. This modified the climate variability, with additional trends at certain intervals and an overall changed frequency spectrum.

Detection and attribution studies aim to quantify the response to external forcings in reconstructions and have shown that particularly volcanism, but also greenhouse gases have a detectable influence on climate reconstructions of the last millennium (Hegerl et al. 2007; Schurer et al. 2013, 2014). However, previous studies have not taken reconstruction method, data availability, or specific proxy biases into account. Here we use pseudoproxy methods to derive fingerprints of external forcings accounting for spectral biases in the proxy reconstructions.

Pseudoproxy experiments (PPEs; Smerdon 2012) have provided valuable insight into effects of reconstruction methods, calibration, coverage, and noise properties on proxy reconstructions. Such experiments involve proxy-network-like data sampling from climate model output and applying proxy methods to derive reconstructions that can be tested in the virtual reality of the model climate. Many pseudoproxy studies have addressed data coverage, location, calibration method, and influences of different noise models (e.g., Von Storch 2004; Bürger et al. 2006; Hegerl et al. 2007; Von Storch et al. 2009; Lee et al. 2008; Christiansen et al. 2009; Neukom et al. 2014). It was found that the addition of noise is one of the most important factors influencing the performance of the different reconstruction methods. Von Storch et al. (2009) showed that adding noise to pseudoproxy data can suppress low-frequency variance of temperature anomalies in the pseudoproxy reconstructions as a consequence of regression during calibration.

In this article, we investigate potential biases in largescale temperature reconstructions that are related to biological effects in tree-ring proxies. First, we introduce our temperature datasets (section 2), followed by methods for pseudoproxy experiments, data analyses, and detection and attribution in section 3. Our results are shown in section 4 , where we compare the spectral properties of observational and proxy data to find a suitable statistical model for pseudoproxy experiments. Based on this, we focus on suitable memory models and evaluate the performance of pseudoproxy reconstructions. Last, we analyze their implications on detection and attribution analyses. We discuss our results in section 5 .

\section{Data}

\section{a. Tree-ring data}

We use tree-ring data provided by the Northern Hemisphere Tree-Ring Network Development (N-TREND) consortium as published by Wilson et al. (2016) and Anchukaitis et al. (2017). This consortium is the result of a collective strategy by the dendroclimatology community to improve large-scale summer temperature reconstructions. The dataset consists of 54 tree-ring chronologies and local reconstructions, which are selected from previously published reconstructions (Table S1 in the online supplemental material). Thus, the data include informed judgments of the original authors for the most robust temperature estimates for each particular location. The individual records use different tree-ring parameters as temperature proxies, including 11 records derived from RW, 18 records MXD, and 25 mixed records (MIX). The mixed records consist of combinations of local, regional, and gridpoint reconstructions derived from RW, MXD, and blue intensity (BI) data. BI is a relatively new method to dendroclimatology and provides similar proxy climate information to MXD [see Campbell et al. (2007), Björklund et al. (2014), and Rydval et al. (2014) for more information].

The records cover the midlatitudinal band between $40^{\circ}$ and $75^{\circ} \mathrm{N}$, following the recommendation of Wilson et al. (2016), as trees farther south are more sensitive to multiple climate influences (Fritts 1976; St. George 2014; St. George and Ault 2014; Osborn and Briffa 2000; Franke et al. 2013). The target area is divided into three continental-scale regions (North America, western Eurasia, and eastern Eurasia). Each region has available data covering more than 1000 years, with 23 records extending back to at least AD 978 All records cover the period from 1710 to 1988 . However, the number of available records decreases markedly toward the beginning of the last millennium, and 

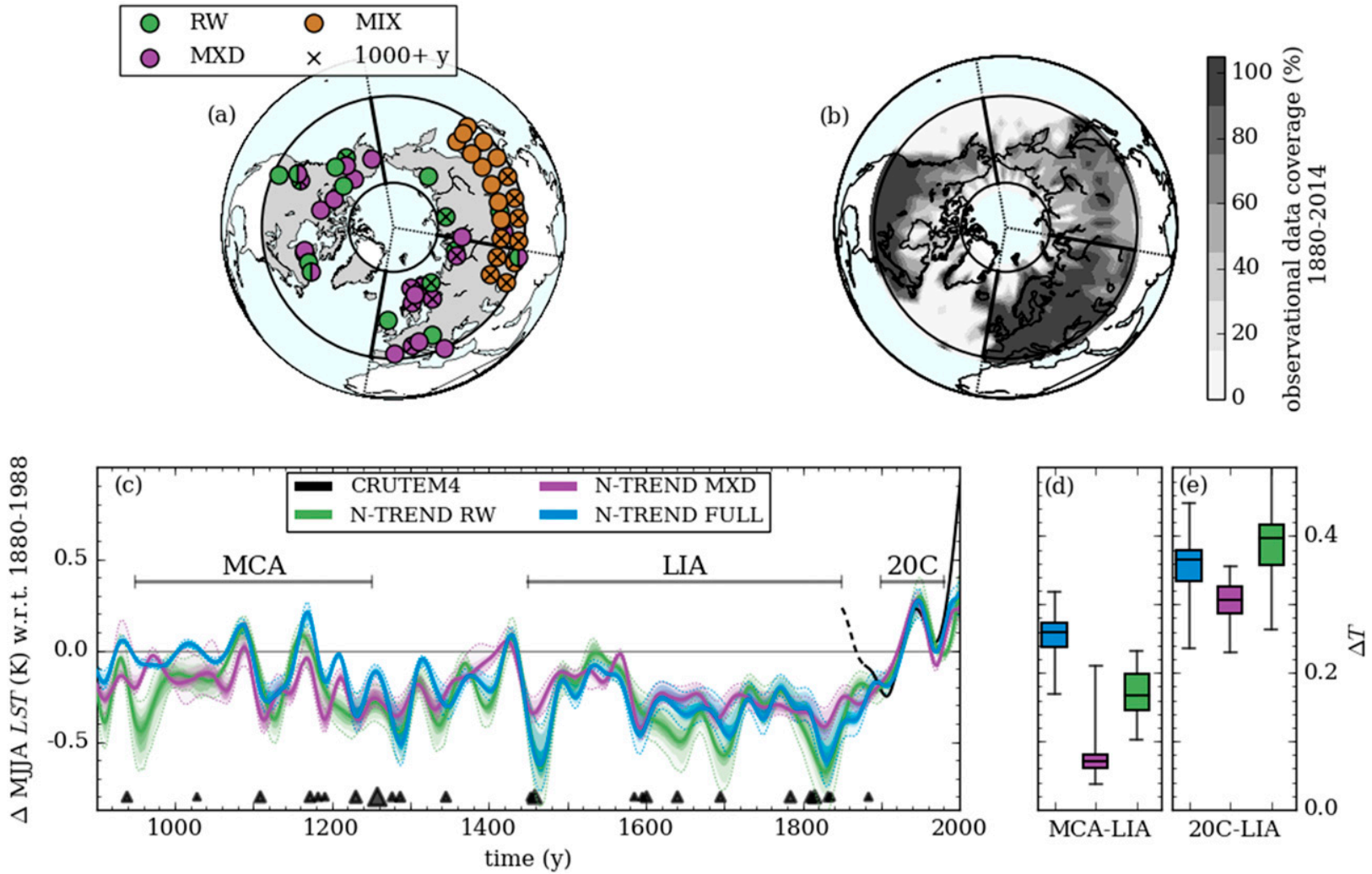

FIG. 1. (a) N-TREND2015 dataset, showing the locations of records derived from ring-width (RW), latewood density (MXD), and combinations of different tree-ring parameters (MIX); $\times$ denotes records longer than 1000 years. (b) Percentage of instrumental data coverage between 1880 and 2014 within the reconstruction target area. (c) FULL, RW, and MXD reconstruction ensembles. The median is shown as a solid line, with the 5th-95th percentiles indicated by a thin dotted line. Shading indicates the 5th-95th percentiles. Instrumental data prior to 1880 are excluded from the analysis due to high uncertainty (dashed). All time series were smoothed using a 20-yr smoothing spline for visualization purposes. Triangles indicate years of volcanic activity and are scaled according to eruption magnitude (Toohey and Sigl 2017). (d) Difference of average temperature of Medieval Climate Anomaly (MCA; 950-1250) and Little Ice Age (LIA; 1450-1850) and (e) twentieth century (20C; 1900-80) and LIA. Boxes range from the upper to the lower quartiles, whiskers indicate the 5 th-95th percentiles, and the solid line is the median.

North America relies on only three records before AD 1100. The individual proxy locations are shown in Fig. 1a.

To understand the effects of different proxy types, we slightly modify the original N-TREND dataset. We distinguish three datasets, consisting of the full network (referred to as N-TREND FULL), RW data only (N-TREND RW), and MXD records only (N-TREND MXD). Given the small number of BI data in the mixed records, we exclude BI-specific biases from our analysis by removing BI data from six mixed records for which the individual records were available. From those mixed records we additionally recover the original RW and MXD chronologies and include them into N-TREND RW and N-TREND MXD to increase the size of the datasets. Table S2 lists the affected sites and which data type was extracted for the different proxy datasets. Hence, the N-TREND MXD dataset consists of
22 tree-ring records in total, while N-TREND RW consists of 17 records.

\section{b. Instrumental data}

The Climatic Research Unit Temperature (CRUTEM4) dataset (Osborn 2013) provides instrumental data over the period from 1850 to 2013. CRUTEM4 is a gridded dataset of global historical near-surface air temperature anomalies over land with a resolution of $5^{\circ}$. The coverage of the reconstruction target area varies and is highly depended on the location (Fig. 1b). Prior to 1880, coverage is largely restricted to western Europe and lower latitudes of eastern North America. In addition to poor coverage, warm biases might arise from poorly shielded instruments for early instrumental data prior to the widespread use of the Stevenson screen (Parker 1994; Böhm et al. 2010; Frank et al. 2007). Given the greater uncertainty (Brohan et al. 2006) and poor data 
coverage, data prior to 1880 were excluded from the analysis. Even at later times, the hemispheric reconstruction is clearly biased toward Europe, where we find many of the grid points covering the full calibration period. North America is well covered at lower latitudes in this period but lacks data at higher latitudes. Coverage is worst for Asia, where most grid points do not start before 1950. This makes the early instrumental record for Asia particularly prone to biases and shifts the hemispheric record heavily to Europe and North America.

\section{c. Climate model data}

We used the Community Earth System Model Last Millennium Ensemble Project (CESM-LME; OttoBliesner et al. 2016) for all model-proxy comparisons and pseudoproxy experiments. The CESM-LME uses a version of CESM-CAM5_CN $(1.9 \times 2.5$ _gx1v6), with a resolution of $\sim 2^{\circ}$ in atmosphere and land components and $\sim 1^{\circ}$ resolution in ocean and sea ice components. External forcings include volcanic, solar, orbital, changes in land use/land cover, and greenhouse gas forcing. Forcing reconstructions follow the recommendations by the Paleoclimate Intercomparison Project Phase III (PMIP3; Braconnot et al. 2012; Schmidt et al. 2011, 2012) and are the same as used in the last millennium simulation of the Community Climate System Model version 4 (CCSM4; Landrum et al. 2013). The CESM-LME provides a large range of different experiments, including all transient forcings as well as ensembles of individual forcings and control runs, covering the period from 850 to 2006. For our analyses, we use an ensemble of 13 climate simulations including all forcings, 5 simulations including volcanic forcing only, and 2 control simulations. To improve like-for-like comparison of model and proxy data, we use only May-August (MJJA) surface temperature data over land and within the N-TREND target area of $40^{\circ}-75^{\circ} \mathrm{N}$.

\section{Methods}

\section{a. Reconstruction method}

Our reconstruction method mostly follows the method introduced along with the original tree-ring dataset (Wilson et al. 2016, 2007; D'Arrigo et al. 2006), targeting Northern Hemispheric (NH) midlatitudinal summer (MJJA) land surface temperature. We first standardize all data to $z$ scores (mean $\mu=0$, variance $\sigma^{2}=1$ ) over the period $1750-1950$, then apply a nesting approach to ensure that the variance is independent of the number of available records (Cook et al. 2002;
Meko 1997). We classify the data into forward and backward nests of common data availability. We define the most replicated nest (NEST1), which includes all records and covers the period $1710-1988$. We then find the other nests by going backward/forward in time and iteratively remove shorter records. A detailed list of the forward and backward nests is given in the supplemental material.

For each nest, we calculate regionally averaged time series. To ensure even contribution from all regions we restandardize the regional time series over the period 1750-1950. The regions are defined as longitudinal slices of the hemispheric band as shown in Fig. 1, providing a time series for North America $\left(170^{\circ}-10^{\circ} \mathrm{W}\right)$, western Eurasia $\left(10^{\circ} \mathrm{W}-80^{\circ} \mathrm{E}\right)$, and eastern Eurasia $\left(80^{\circ} \mathrm{E}-\right.$ $\left.170^{\circ} \mathrm{W}\right)$. This approach slightly differs from the original method, in which North America had been additionally divided along the meridian at $100^{\circ} \mathrm{W}$. By doing so, we ensure that more data are available for each region. This is important when constructing time series for RW or MXD only, which further reduces the number of available proxy records.

We derive a hemispheric mean series $z_{i}(t)$ for each nest $i$ by averaging over the regional time series and calibrate the result for NEST1 $z_{1}(t)$ to the instrumental data $T_{\text {obs }}(t)$. The calibration covers the period 1880-1988. We choose the start date to exclude poor instrumental coverage and the end date to ensure full coverage by the tree-ring network. Calibration includes matching of variance and mean (Esper et al. 2005) of instrumental and proxy data:

$$
T_{1}(t)=z_{1}(t) \times \sigma_{\mathrm{obs}}^{2}+\mu_{\mathrm{obs}}
$$

The hemispheric time series from all other nests are scaled to $T_{1}(t)$, the temperature time series obtained from NEST1, in the same way but each over the full period of NEST1. Ultimately, a homogeneous temperature reconstruction is derived by extracting the temperature for each year from the densest nest available. Comparing the different proxy datasets (Fig. 1c) we find that long- and short-term variability varies across the datasets, with FULL and RW displaying more lowfrequency variability throughout the last millennium. This is highlighted in the average temperature difference between the Medieval Climate Anomaly (MCA, 950-1250; Masson-Delmotte et al. 2013) and Little Ice Age (LIA, 1450-1850; Masson-Delmotte et al. 2013). MXD shows a smaller difference than RW and FULL. This can also be observed when comparing differences between twentieth-century warming and LIA, which is consistently higher in RW than in MXD data. As discussed by Wilson et al. (2016), the N-TREND 
reconstruction shows little divergence (Wilson et al. 2007; D'Arrigo et al. 2008) from the instrumental data during the late twentieth century. However, to exclude potential influences of the remaining divergence we use the period $1900-80$ as representative for twentiethcentury warming. All proxy reconstructions show a similar temperature difference between the LIA and this period.

\section{b. Reconstruction uncertainty}

Quantifying and including all forms of uncertainty in tree-ring (and other proxy) climate reconstructions is a significant challenge and beyond the scope of this article. However, we can model uncertainties caused specifically by coverage and calibration relatively easily using an ensemble approach (Frank et al. 2010b; Neukom et al. 2019). To be able to replicate the same reconstruction method when conducting our pseudoproxy experiments, it was important to reduce computational time and thus keep the ensemble size relatively small. To address the coverage uncertainty, we apply a bootstrapping approach to the proxy dataset, in which one proxy record is removed in turn before creating the reconstruction. Although this would ideally include the removal of each proxy record in the dataset in turn, we restrict the analysis to bootstrapping nine randomly selected long records in turn, extending back to at least AD 1150. Thus, we estimate the coverage uncertainty specifically in the poorly covered periods. The chronologies that were in turn removed from N-TREND FULL were AG12, AG4, FORF, AG2, ALT, AG5, AG1, AG11, and FIRT. For MXD they were ALT, POLx, JAEM, ALPS, FORF, TYR, FIRT, ICE, and SFIN. For RW they were TAT, KOL, QUEw, OZN, GOA, ICE, YAM, IDA, and TAY. (For descriptions of all the chronologies, see the online supplemental material.) Including the set consisting of all available records, we gain a total ensemble of 10 sets of data for each N-TREND dataset, consisting of $1 \times 54+9 \times 53$ records for N-TREND FULL, $1 \times 22+9 \times 21$ for MXD, and $1 \times 17+9 \times 16$ for RW.

To address the calibration uncertainty, we slice the calibration period into windows of lengths 60,70 , and 80 years similar to Frank et al. (2010b). For each window length, we perform the calibration for an early, a middle, and a late period (1880-1940, 1904-64, 1928-88, 18801950, 1899-1969, 1918-88, 1880-1960, 1894-1974, and 1908-88). Including the full period, we thus consider 10 different implementations of calibration periods, gaining a total reconstruction ensemble of 100 reconstructions for each N-TREND dataset (FULL, RW, and MXD). This allows us to estimate the spread of our results depending on calibration and coverage uncertainty.

\section{c. Pseudoproxy experiments}

For our PPEs, we generate sets of pseudoproxy data from climate model output and treat them in the same way as real proxy data. We sample from the CESMLME ensemble at the grid cells closest to the proxy record to match spatial and temporal availability of the N-TREND dataset as in Neukom et al. (2018). For proxy records that represent an area larger than a single grid point, the average over all grid cells within the target area was calculated. The same was repeated for CRUTEM4 to generate a pseudoinstrumental dataset. The pseudoproxy data were then processed in the same way as the real proxy reconstruction, including standardizing $(\mu=0, \sigma=1)$, nesting, regional averaging, calibrating to the pseudoinstrumental dataset and splicing of the nested data to obtain a hemispheric pseudoreconstruction. To account for calibration and coverage uncertainty, the calibration period was varied, and longer records were bootstrapped in the same way as in the case of the real proxies. The same periods and chronologies as detailed in section $3 \mathrm{~b}$ were used to create a total ensemble of 1300 PPEs from the 13 CESM LME simulations and 500 PPEs from the 5 volcanic-forcing-only simulations.

Thus, the pseudoproxy reconstruction represents the spatiotemporal availability of the proxy network and reconstruction methods; however, it does not account for any proxy specific biases or nonclimatic influences. This PPE serves as the baseline to represent characteristics of local climate model data without simulating tree-ring memory. It is referred to as PPE NoM. To simulate biological-based memory we manipulate the pseudoproxy records at the local scale. Two different memory models were distinguished: a short-range autoregressive model of order $p$ (PPE AR) and a long-term memory (LTM) model (PPE LTM). To concentrate on the effects of memory, we have not added additional nonclimatic white noise to the pseudoproxies. An overview of the different experiments, their ensemble sizes and fitting parameters is given in Table 1.

\section{1) PPE AR}

This memory model is based on a linear decomposition of the tree-ring signal $z$ into a climate term and an autoregressive memory term of order $p$. The tree-ring signal $z_{t}$ of a given year $t$ is impacted by the locally modeled climate signal $x_{t}$. This signal is subjected to a memory term, which integrates over the previous $p$ year's signals $z_{t-1}, z_{t-2}, \ldots z_{t-p}$. The signal at time $t$ can thus be written as

$$
z_{t}=x_{t}+\sum_{k=1}^{p} \alpha_{k} z_{t-k}+\varepsilon_{t}
$$


TABLE 1. Ensemble sizes for N-TREND and PPEs, each applied to the FULL, RW, and MXD target dataset.

\begin{tabular}{|c|c|c|c|c|c|}
\hline Name & Fitting parameter & Calibration & Coverage & Simulations & Total \\
\hline N-TREND & - & $1+9$ & $1+9$ & - & 100 \\
\hline PPE NoM & - & $1+9$ & $1+9$ & 13 & 1300 \\
\hline PPE AR3 & $\alpha_{1}, \alpha_{2}, \alpha_{3}$ & $1+9$ & $1+9$ & 13 & 1300 \\
\hline PPE LTM & $\beta$ & $1+9$ & $1+9$ & 13 & 1300 \\
\hline PPE NoM- VOLC & - & $1+9$ & $1+9$ & 5 & 500 \\
\hline PPE AR3-VOLC & $\alpha_{1}, \alpha_{2}, \alpha_{3}$ & $1+9$ & $1+9$ & 13 & 500 \\
\hline PPE LTM- VOLC & $\beta$ & $1+9$ & $1+9$ & 13 & 500 \\
\hline PPE NoM- CTRL & - & $1+9$ & $1+9$ & 2 & 200 \\
\hline PPE AR3- CTRL & $\alpha_{1}, \alpha_{2}, \alpha_{3}$ & $1+9$ & $1+9$ & 2 & 200 \\
\hline PPE LTM- CTRL & $\beta$ & $1+9$ & $1+9$ & 2 & 200 \\
\hline
\end{tabular}

$$
=\sum_{k=1}^{q} \gamma_{k} x_{t-k}+\sum_{k=1}^{p} \alpha_{k} z_{t-k}+\varepsilon_{t}
$$

where $\varepsilon_{t}$ accounts for additional white noise. The set of parameters $\alpha$ determines the influence of the $k$ previous years' climate on the proxy signal and represents the memory term. The first term represents the climate forcing, which accounts for the autoregressive structure of the climate signal $x_{t}$ itself. The autoregressive character of the climate is parameterized by the coefficients $\gamma$ and its order $q$. If $x_{t}$ represents a zero-mean white noise process, Eq. (3) represents an autoregressive moving-average process $[\operatorname{ARMA}(p, q)]$. This is an autoregressive process of order $p$ forced by a movingaverage process of order $q$ (Box 2016; Von Storch and Zwiers 2002). Assuming the climate signals of the model simulations perfectly match the real world, the climate signal $x_{t}$ is given by the model data, averaged over the proxy target area. With the starting points of the time series fixed up to $x_{p}, z_{i>p}$, can be iteratively calculated if the memory parameters $\alpha_{j}$ are known. Instead of fitting an $\operatorname{ARMA}(p, q)$ process with $p+q+2$ degrees of freedom on the proxy data, we apply an empirical approach for fitting the memory. We use the knowledge of the model climate signal $x$ and the proxy signal $z$ to find an estimate for $\alpha_{k}$, which produces pseudoproxies with a similar memory as seen in the proxy records.

To identify the autoregressive structure in proxy records $z$ and model $x$, the partial autocorrelation function (PACF) was calculated. The PACF $\phi_{k}$ of a time series $y$ at lag $k$ determines the correlation between $y_{t}$ and $y_{t-k}$, which is not accounted for by $y(t-1), \ldots, y(t-k+1)$. Given that the partial autocorrelation of an $\operatorname{AR}(p)$ process decays to zero beyond lag $p$ we can use it to identify the order $p$. The coefficients $\phi_{i}$ can be calculated from the Yule-Walker equations (Box 2016). An initial estimate for the memory coefficients $\alpha$ was obtained by using

$$
\alpha_{k}=\phi_{k}(z)-\phi_{k}(x),
$$

with the PACF $\phi_{k}(z)$ and $\phi_{k}(x)$ at lag $k$ for the proxy record $z$ and the targeted model data $x$. This was found to be a good estimate for all lags higher than lag 1. For lag $1, \alpha$ was systematically overestimated by Eq. (4), therefore an optimization algorithm was implemented to fit the PPE to the proxy target value.

A set of fitting parameters was derived for each proxy record $z$ in the target dataset, and the associated pseudoproxy record $\tilde{z}$ was fitted using Eq. (2). We set $\varepsilon=0$, concentrating on the effects of pure memory addition. To determine whether the results are spatially robust, we randomly redistributed the parameters $\alpha$ over the pseudoproxy locations. We found that the spread of results is minimal compared to the spread caused by the variation of the calibration period and bootstrapping. To keep the ensemble number at a reasonable size we therefore did not include this uncertainty into the final ensemble of PPEs.

\section{2) PPE LTM}

This method involves a manipulation of the time series in its Fourier space, which is based on a previously published study by Zhang et al. (2015). For a time series possessing LTM, its power spectral density will decay with

$$
S(f) \sim f^{-\beta} .
$$

The parameter $\beta$ is a measure of the long-term memory. For white noise processes $\beta \approx 0$, whereas for red noise $\beta=2$. A robust estimate for $\beta$ can be obtained from a detrended fluctuation analysis of the second order (DFA-2) (Peng et al. 1994; Bryce and Sprague 2012). For a time series $x(t)$ with zero mean $\langle x\rangle$ the cumulative sum $X_{t}=\sum_{i=1}^{t}\left(x_{i}-\langle x\rangle\right)$ is divided into $N$ segments with window length $n$. The local trend $Y_{t}$ for each segment is derived from a least squares quadratic fit of $X_{t}$. The 
root-mean-square deviation of $X_{t}$ from the local trend for any window-length $n$ gives the fluctuation function

$$
F(n)=\sqrt{\frac{1}{N} \sum_{t=1}^{N}\left(X_{t}-Y_{t}\right)^{2}} .
$$

If $F(n)$ follows a power-law scaling $F(n) \sim n^{\alpha}$, the spectral density will satisfy Eq. (5) and

$$
\beta=2 \alpha-1 \text {. }
$$

A double logarithmic plot of the fluctuation function can provide information about the amount of LTM in a time series and a robust estimate for $\alpha$ can be calculated from a linear fit.

It was shown in previous studies that surface temperature follows a slight LTM process on both hemispheric and regional scales (e.g., Rypdal and Rypdal 2014), with $\beta \approx 0.2$ at regional scale and $\beta \approx$ 0.4 over land (Fredriksen and Rypdal 2016). Assuming that biological tree-ring memory $y(t)$ can be represented by an LTM process that is superposed on the climate signal $x(t)$, its spectral energy can be approximated as

$$
S_{z}(f)=S_{0}(f) f^{\beta_{z}} \approx S_{x}(f) f^{\beta_{y}}=S_{0}(f) f^{\beta_{x}+\beta_{y}} .
$$

The factor $S_{0}(f)$ accounts for the remaining signal and represents a white noise process. Equation (8) is linear in $\beta$, which can be used to estimate the additional memory $\beta_{y}$ and fit the pseudoproxy records

$$
\tilde{S}(f)=S(f) \beta_{y}, \quad \beta_{y}=\beta_{z}-\beta_{x} .
$$

This way a pseudoproxy record with energy spectral density $S(f)$ is fitted such that its LTM is increased to proxy level. The inverse Fourier transform of the manipulated record $\tilde{S}(f)$ gives the pseudoproxy record $\tilde{z}(t)$.

\section{d. Superposed epoch analysis}

A superposed epoch analysis is used to reveal the response to volcanic forcing evident in last millennium temperature reconstructions (e.g., Lough and Fritts 1987; Mass and Portman 1989; Hegerl et al. 2003; D'Arrigo et al. 2013; Masson-Delmotte et al. 2013; Esper et al. 2015; Wilson et al. 2016; Neukom et al. 2018). We average over the temperature response to a set of volcanic eruptions, using a window of maximally 30 years, considering temperature anomalies with respect to 10 years preceding a volcanic eruption. Any subsequent years within the recovery time of an event that are affected by major eruptions are excluded from the epoch analysis.
We assume that the latest reconstruction of atmospheric sulfate injection (eVolv2k) as published by Toohey and Sigl (2017) minimizes the dating error for the proxy reconstructions. The volcanic forcing dataset implemented in the CESM-LME is based on the IVI2 reconstruction by Gao et al. (2008). Both datasets are based on ice core data and provide a measure of aerosol optical depth (AOD) and stratospheric sulfate injection. However, dating and magnitude of volcanic eruptions in IVI2 differ in many cases from eVolv2k. To perform a like-for-like comparison, we therefore use eruption dates as given in eVolv $2 k$ for the proxy data, while using IVI2 dates for the model/PPE data. To increase the number of events while minimizing the error induced by dating uncertainty, we consider only events that appear within 3 years of difference in both datasets. The 16 events included in the epoch analysis have been marked. Note that the eruptions in 1761-62 and 1783 (Laki) were excluded from the analysis despite matching dating. As noted in Stevenson et al. (2017) in the CESM-LME Laki is wrongly dated at 1761 instead of 1783, which makes both dates unsuitable for our comparison. A table showing all eruptions is given in the online supplement. It should also be noted that the dating of volcanic eruptions in the climate model/PPEs follows exactly IVI2 and thus has no dating uncertainty. However, due to the uncertainty in the icecore-based reconstructions of volcanic forcing, some degree of dating uncertainty remains in the analysis of the tree-ring data. Nevertheless, we assume that with our approach we have kept the dating uncertainty minimal.

\section{e. Detection and attribution studies}

To quantify the influence of forced variability in the proxy reconstructions, we perform detection and attribution using a total least squares (TLS) regression following (Stott et al. 2001; Allen and Tett 1999). The proxy reconstruction $Y(t)$ is regressed onto the fingerprint of volcanic forcing $X_{1}(t)$ and all other forcings $X_{2}(t)$, following

$Y(t)=\beta_{1} \times\left[X_{1}(t)-\nu_{1}(t)\right]+\beta_{2} \times\left[X_{2}(t)-\nu_{2}(t)\right]+\nu_{0}(t)$.

The fingerprints of external forcing are given by the simulations of the CESM-LME. A TLS regression allows regressor $X(t)$ and regressand $Y(t)$ to be influenced by a similar amount of noise, which is given by their respective implementation of internal variability $\nu(t)$. The amount of internal variability in the fingerprints $X(t)$ can be reduced by averaging over multiple ensemble members. The scaling factors $\beta_{i}$ indicate the 

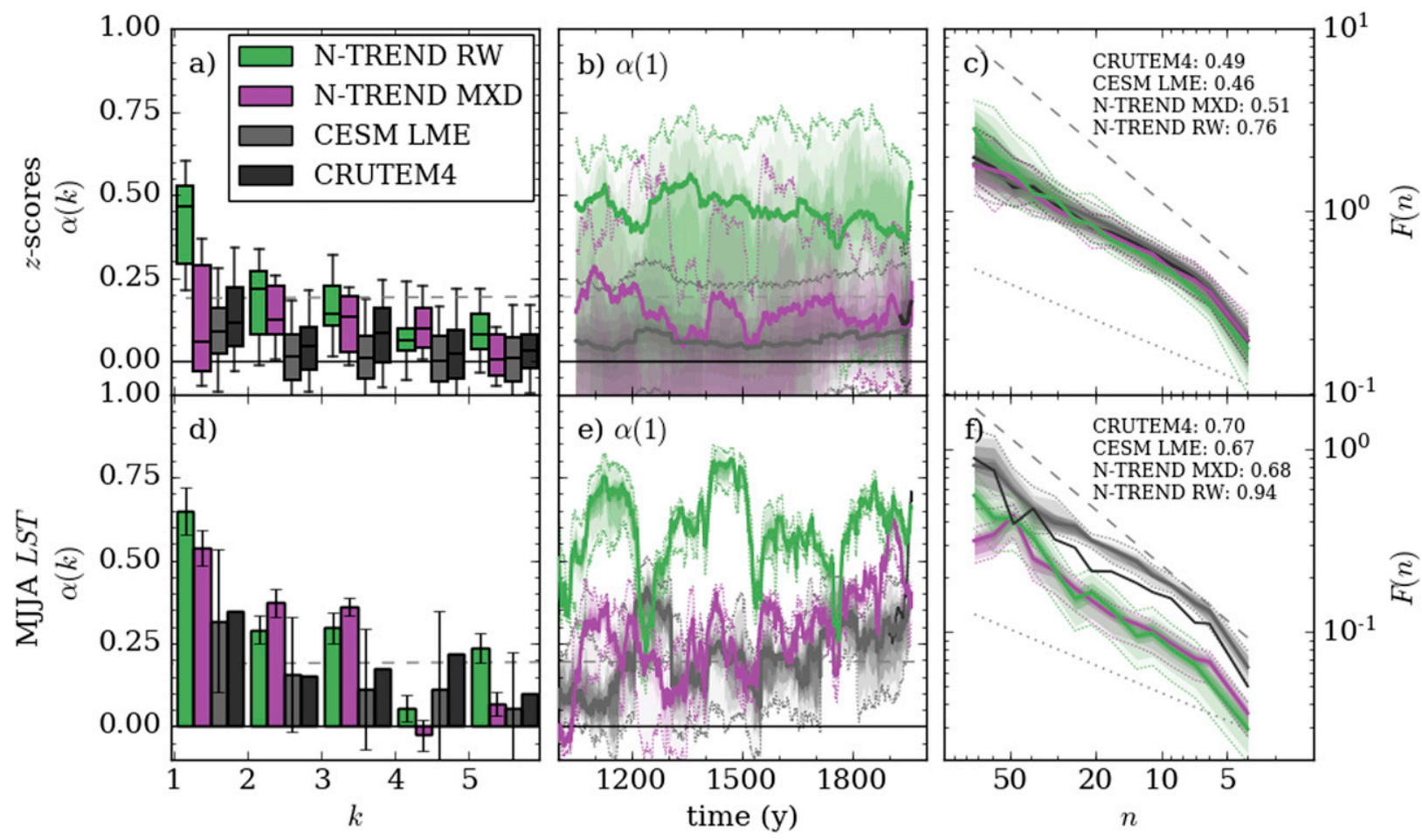

FIG. 2. (a) Partial autocorrelation (PACF) $\alpha(k)$ during the calibration period (1880-1988) for local standardized records ( $z$ scores). Box: upper to lower quartiles; whiskers: 5th-95th percentiles; line: median. (b) Median of PACF at lag 1 and percentile range (shaded) of the $z$ scores, calculated over a centered 100-yr sliding window during the last millennium (1000-2000). (c) Detrended fluctuation analysis of the $z$ scores during the calibration period. Dotted (dashed) lines indicate the gradient expected for white (pink) noise. (d)-(f) As (a)-(c), but for the mean of hemispheric temperature reconstructions. Bars in (d) indicate the 5th-95th percentiles of the ensembles. Note that the CESM includes 13 simulations and has a much higher spread accordingly.

magnitude of the fingerprints in the reconstruction. The response to a forcing is considered detectable $(p<0.05)$ when the scaling factor is significantly positive. A scaling factor of 1 indicates perfect agreement between models and proxy reconstruction (Hegerl and Zwiers 2011). The residual gives an estimate of internal variability in the proxies. To account for the uncertainty due to internal variability and to get a distribution for the scaling factors, we follow the method introduced by Schurer et al. (2013, 2014). We repeated our calculations 100 times with different samples of internal variability superimposed on the noise-reduced observations and model fingerprints $\tilde{Z}=\left[Y(t)-\nu_{0}(t), X_{i}(t)-\nu_{i}(t)\right]$. To investigate the effects of autocorrelation in proxy data on detection and attribution results, we further repeated our analyses using pseudoproxy fingerprints.

\section{Results}

\section{a. Spectral properties of observations and model simulations compared to tree-ring data}

We compare the spectral characteristics of the proxy datasets to a set of local instrumental and model records over the period 1880-1988. This period provides the maximum availability for the proxy data and is well covered by the instrumental dataset.

For the PACF at local scale (Fig. 2a), the biggest differences can be noted at lag 1 , where RW displays a higher correlation than all other datasets. At all lags, correlation is highest for RW, followed by MXD, replicating the findings of Esper et al. (2015). Model and instrumental data agree well, with observational data showing a slightly higher correlation at all lags. The medians of the PACF at lag 1 differ by $\Delta \alpha \approx 0.4$ for RW and MXD, which remains relatively constant during the period of common data availability (Fig. 2b). N-TREND MXD is slightly higher than the CESM-LME ensemble but is consistent within its 5th-95th-percentile range. MXD also agrees well with the observations within the short period in which instrumental data are available. We compute the detrended fluctuation function for each record (Fig. 2c) to obtain an estimate for the long-term memory at local scale using Eq. (7). Results for all datasets are relatively widely spread but overlap at the 5th-95thpercentile range. The median of MXD, observations 

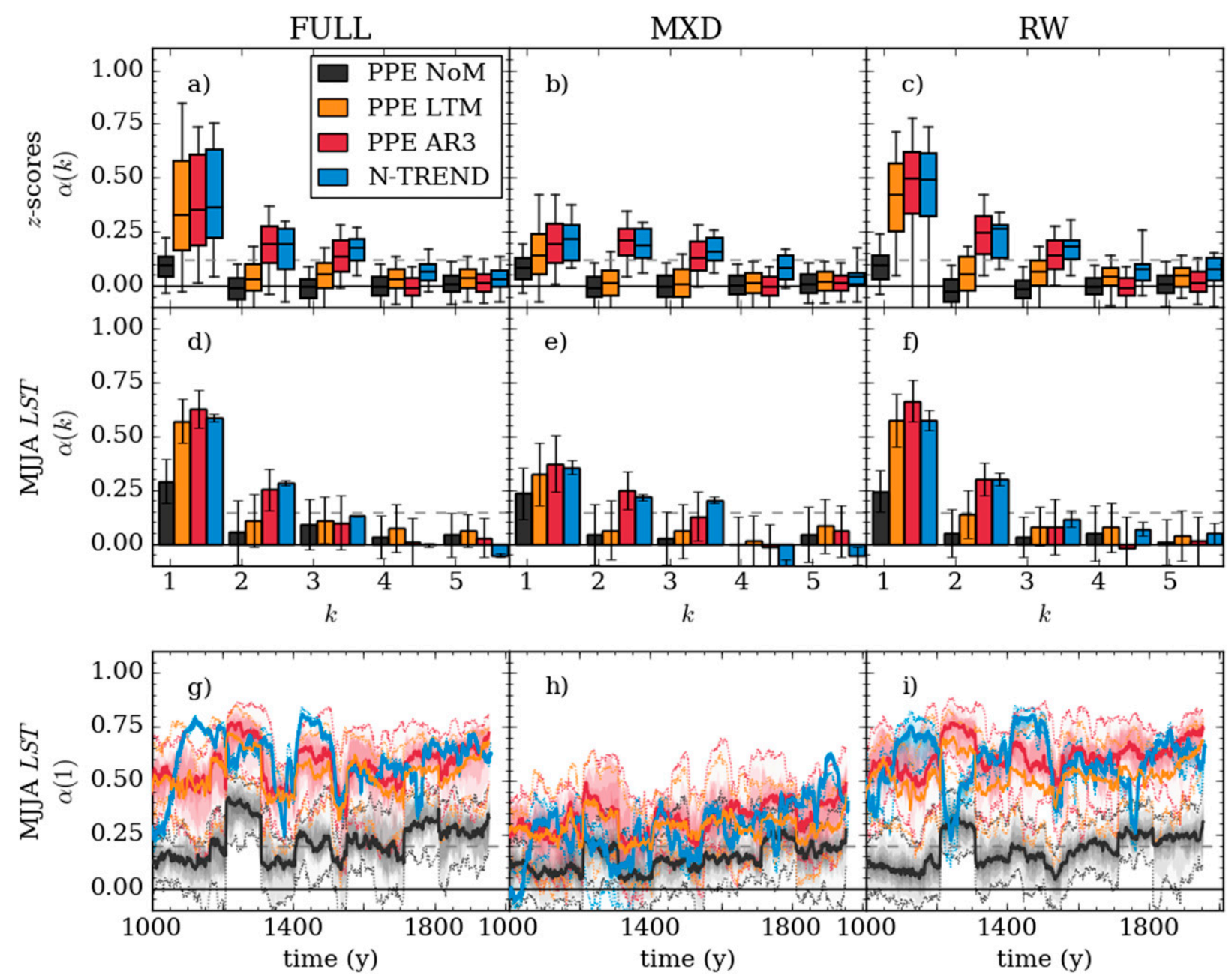

FIG. 3. (a)-(c) PACF between AD 1000 and 1900 for real proxy $z$ scores (N-TREND) and pseudoproxy experiments (PPEs) on a local scale for the full proxy dataset and RW and MXD records only. PPE NoM refers to pseudoproxies from raw model runs, AR3 to pseudoproxies fitted by a third-order autoregressive model, and LTM to the long-term memory fit. (d)-(f) PACF of hemispheric temperature reconstruction for the same period. (g)-(i) The 100-yr running mean of the PACF at lag 1.

and CESM-LME agree with $\beta \approx 0.5$, while $\mathrm{RW}$ proxies have slightly more memory $(\beta \approx 0.8)$.

Results at hemispheric scale are similar and show that the features observed on the local scale propagate into the reconstructions. The PACF (Fig. 2d) is still highest for RW at lag 1 while MXD is more persistent at lags 2 and 3. Modeled and observed temperatures have less PACF at these lags. Note that at lag 4 the PACF is just above the significance level for observational data and some model simulations. It is not clear whether this is a real climatic feature or sampling noise. The magnitude of the lag $1 \mathrm{PACF}$ of the MXD reconstruction agrees well with the model mean (Fig. 2e) but RW correlation is still significantly higher during most of the period of common data availability. The magnitude of fluctuation (Fig. 2f) is similar for RW and MXD; however, RW has more memory with $\beta \approx 0.9$ compared to $\beta \approx 0.7$ for MXD. MXD agrees well with model and instrumental data $(\beta \approx 0.7)$.

Our results suggest that an autoregressive process around order 3 can be fitted to the proxy data. Given that observational and model data seem to follow mainly an order- 1 process, we conclude that the thirdorder process is caused by nonclimatic noise such as biological memory processes.

\section{b. Spectral properties of pseudoproxy data compared to real proxy data}

We generated pseudoproxy data for different memory models, concentrating on an autoregressive process of order 3 (PPE AR3) and a long-term memory fit (PPE LTM). We compare the partial autocorrelation of different pseudoproxy experiments with real proxy data 

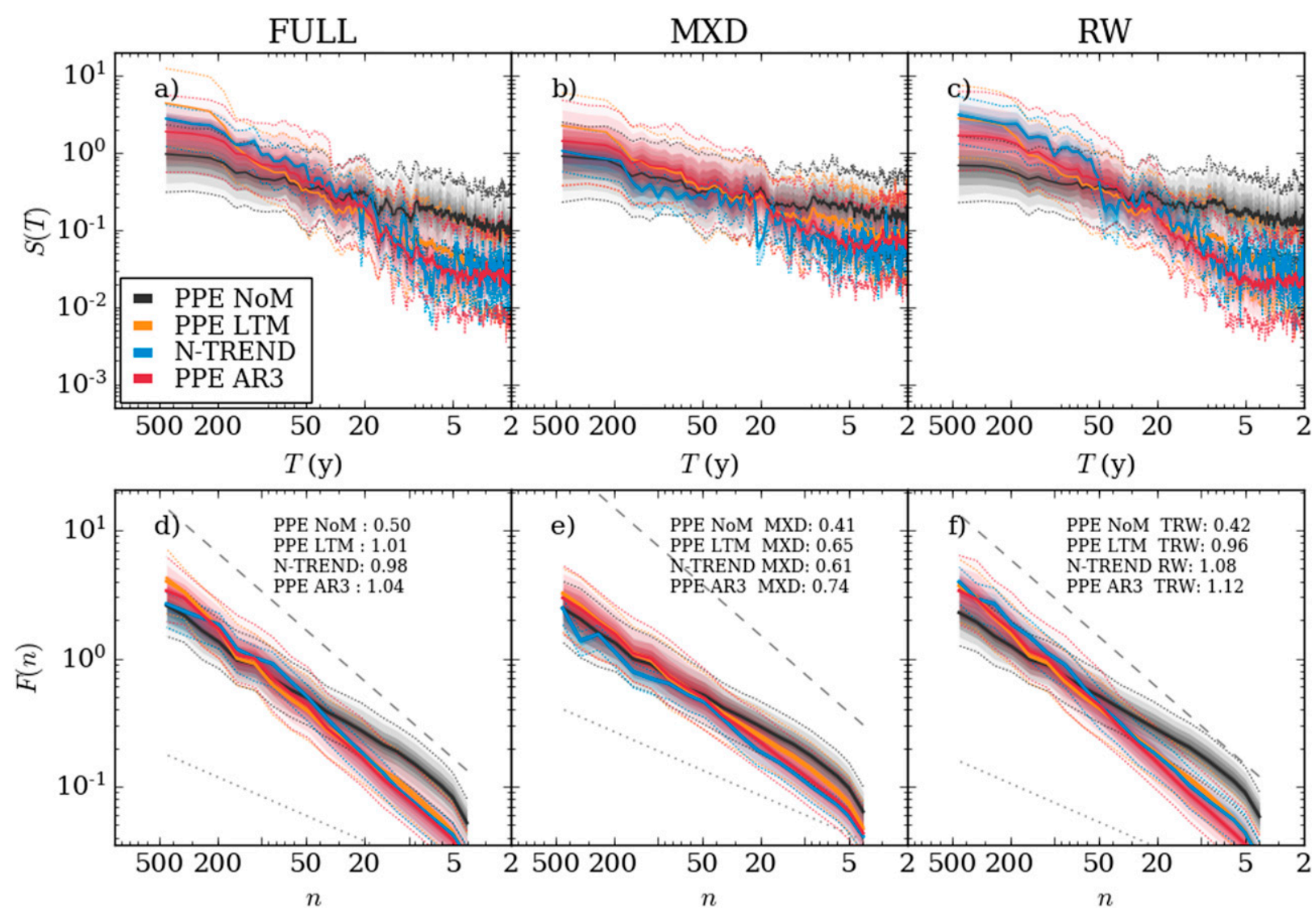

FIG. 4. (a)-(c) Median and percentile range of the power spectral density $S(T)$ of proxy reconstructions compared to the PPEs, with ensemble range for PPE NoM and PPE AR3. The spectrum has been smoothed using a 7-yr running mean filter to increase the visibility of the trend. (d)-(f) Detrended fluctuation analysis $F(n)$ for proxy and pseudoproxy reconstructions. Dotted and dashed lines indicate the gradient displayed by white $(\beta=0)$ and pink noise $(\beta=1)$, respectively.

targeting the full network, MXD only, and RW only. On the local scale (Figs. 3a-c), correlations of PPE NoM are significantly below the range of the correlation for all targets. All pseudoproxy records including memory match the real proxy range at lag 1 . At higher lags, PPE LTM decays quickly below the proxy range while PPE AR3 matches the proxy records even at higher lags. At the hemispheric scale (Figs. 3d-f), differences between PPE AR3 and PPE LTM are smaller but PPE AR3 still performs better. Throughout the last millennium, the lag-1 partial correlation for the pseudoproxies is shifted up to proxy level (Figs. 3g-i) but otherwise barely deviates from PPE NoM.

All the targeted proxy reconstructions have more power at low frequencies than at high frequencies (Figs. 4a-c). The power spectral density follows approximately a power-law decay for multidecadal frequencies, observed as a linear decrease in the double logarithmic plot. However, the gradient flattens toward decadal frequencies, indicating a deviation from the power law. This is particularly prominent in case of RW but can also be observed in the other datasets. The multidecadal gradient is matched by the pseudoproxy reconstructions when accounting for memory, while PPE NoM has a much smaller gradient. PPE AR3 performs well for all targets. It overlaps well with the proxy ensemble within the 5th-95thpercentile range and its median shows the distinctive flattening of the gradient toward its high-frequency end. While PPE LTM also overlaps well with the proxy ensemble within the uncertainty range, the median decreases monotonically. Note that the spectral density of MXD is particularly noisy at low frequencies (Fig. S5). Since this is specific to the MXD dataset, it could be caused by local influences but could also originate from data processing.

The DFA (Figs. 4d-f) confirms that PPE NoM has less long-term memory than the proxies, holding particularly for $\mathrm{RW}(\beta \approx 0.3$ vs $\beta \approx 0.9)$ and FULL $(\beta \approx 0.4$ vs $\beta \approx 0.8$ ), while the difference is smaller in case of MXD $(\beta \approx 0.3$ vs $\beta \approx 0.6)$. PPE AR3 and PPE LTM both replicate the gradient of the proxy targets. While for RW and FULL the average of PPE AR3 and the proxy target overlap roughly for most time steps, the 

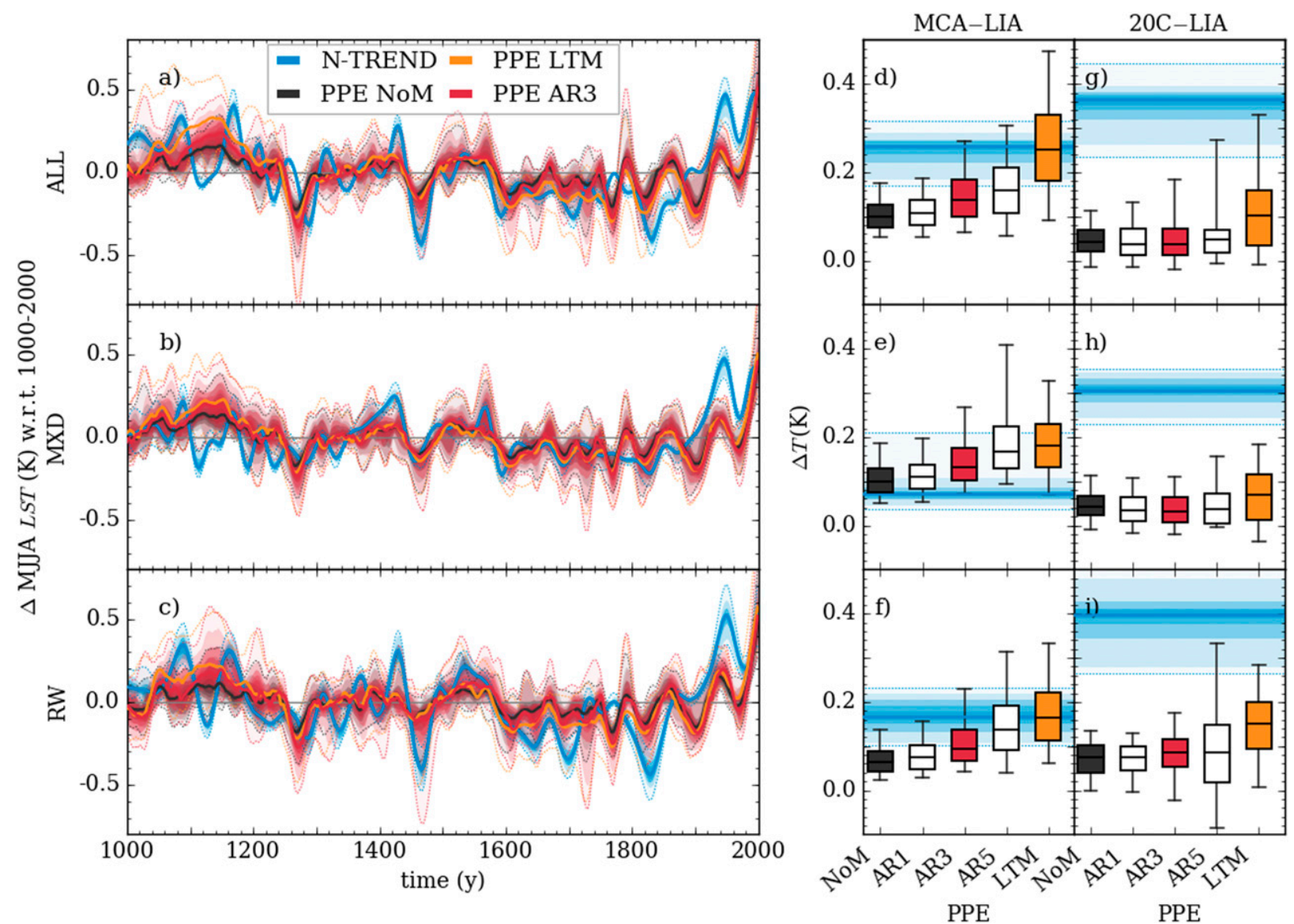

FIG. 5. (a)-(c) Reconstructions of temperature anomalies during the last millennium displayed by real proxies and PPEs. Shading is as in previous figures. (d)-(f) Difference between average temperature of Medieval Climate Anomaly (MCA; 950-1250) and Little Ice Age (LIA; 1450-1850). (g)-(i) Difference between the average temperature of the LIA and twentieth century (20C; 1900-80). Blue horizontal lines and shading indicate the median and the percentiles of the proxy reconstruction, respectively. Boxplots are as in previous figures.

magnitude of the fluctuation of the proxies is consistently lower than the PPEs.

We conclude that PPE AR3 and PPE LTM both reproduce spectral features characteristic to proxy data, such as increased autocorrelation at lag 1, inflation (suppression) of low-frequency (high-frequency) variability, and more long-term memory. PPE AR3 performs best for all target datasets as it matches the partial autocorrelation at higher lags and reproduces the deviation of the spectral density from the power-law decay at high frequencies.

\section{c. Effects of memory on temperature variability of pseudoproxy reconstructions}

The ensemble mean and range of the millenniallength time series for the proxy and pseudoproxy reconstructions are shown in Figs. 5a-c. Long-term deviations from the mean are inflated for memory PPEs compared to PPE NoM. As a result, the MCA is warmer for PPE AR3 and PPE LTM, while the LIA is slightly colder. This trend can be observed in all three target datasets but is particularly strong for FULL and RW.

To quantify the effects of this inflation, we calculate the average temperatures of MCA and LIA. The temperature difference between those periods ranges around $\Delta T=0.2$ for FULL and RW but is less than half for MXD (Fig. 5e). However, the uncertainty on the exact value is relatively high due to the small number of available records at early times. Schneider et al. (2015) found that the MCA is less pronounced in MXD data, suggesting varying seasonal or spatial coverage as a reason. However, PPE NoM shows a clear warming in the MCA for the MXD locations. For all target datasets, the median of $\Delta T$ is increased when implementing memory in the pseudoproxies. For PPE AR3 the median shifts toward the proxy value in case of FULL and RW targets. The temperature difference increases further for higher memory, with PPE LTM consistently being highest. The increase of $\Delta T$ with memory order is a robust feature, which can also be seen when 
comparing average temperatures of the LIA and the twentieth century between 1900 and 1980 (Figs. 5g-i). Note that twentieth-century warming is slightly underestimated in the CESM-LME, likely due to strong indirect aerosol forcing (Otto-Bliesner et al. 2016). This could be a reason for a small temperature difference compared to the proxy value and could suppress stronger increase for memory PPEs.

To analyze the effects of biological memory on the magnitude and time scales of cooling in response to volcanic eruptions (Fig. 6), we perform a superposed epoch analysis (Figs. 7a-c) including 16 well-dated volcanic eruptions. Schneider et al. (2015) compared the volcanic response in a density only reconstruction to ring width dominated reconstructions for the eruptions in 1257, 1452, and 1815. They found that the former shows a greater response amplitude, while the latter show a temporally extended cooling and thus a longer recovery period. The same observations hold for our epoch analysis. Here, MXD responds strongly and recovers fast, with a slightly prolonged cooling around years 3-5. RW has a smaller amplitude along with a prolonged cooling up to posteruption year 10. While the magnitude of the PPE NoM amplitude varies slightly across the target datasets, it recovers much quicker than the proxies. Both magnitude and recovery time are affected by autoregressive memory, most prominently for RW, while long-term memory mainly dampens the amplitude. PPE AR3 shows a prolonged cooling, which is mostly consistent with the time scale of the proxy data. The median of the peak response of the PPE AR3 ensemble is much dampened compared to PPE NoM, and even slightly lower than N-TREND. However, it is consistent with N-TREND within the 5th-95th-percentile range.

Comparing the residuals of proxy and PPE epoch analysis (Figs. S2a-c), we note that the residuals increase particularly between year 3 and year 5 after the eruption. This observation holds for all PPE's and for all target datasets. To increase our understanding, we compare an ensemble member of the CESM showing a particularly prolonged recovery and persistent cooling in year 4 after the eruption (Figs. 7d-f) and one with a particularly quick and steadily decreasing recovery (Figs. 7g-i). In the former case, PPE AR3 reproduces the recovery time, the peak cooling and overlaps with N-TREND for all datasets within its uncertainty range. The residuals are negligibly small 5 years after the eruption (Figs. S2d-f). In the latter case, even though the cooling is more prolonged for PPE AR3 compared to PPE NoM neither its recovery time nor its amplitude match the proxy amplitude. The residuals are near constant up to year 15 (Figs. S2g-i). We

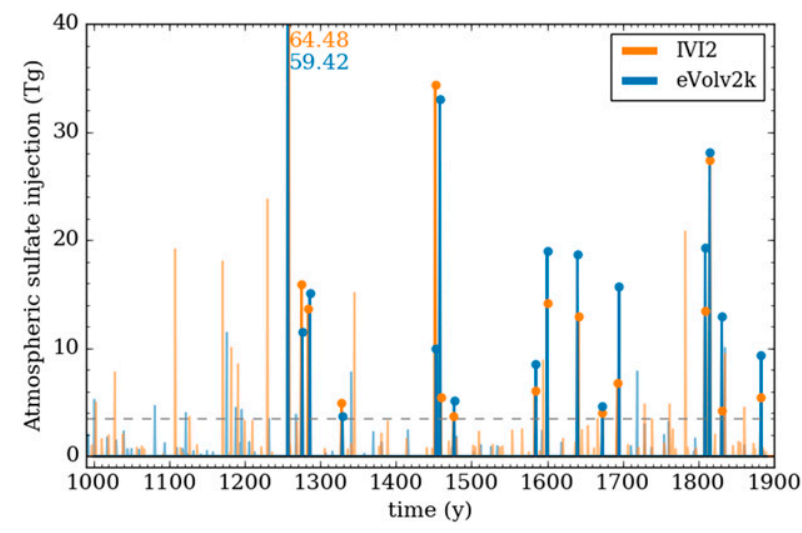

FIG. 6. Overview over atmospheric sulfate injection in IVI2 (Gao et al. 2008) and eVolv2k (Toohey and Sigl 2017). Events chosen for the proxy (PPE) epoch analysis are highlighted and marked by a blue (orange) dot.

conclude that model and proxy output can be consistent when taking memory effects into account. Memory can explain the long recovery time observed in proxy reconstructions but requires persistent cooling on a time scale between 3 and 5 years. This short-term persistence could be caused by internal variability, but also by missing short-term feedback mechanisms in the model, for example, changes in the North Atlantic Oscillation (Zanchettin et al. 2013; Driscoll et al. 2012; Timmreck 2012).

\section{d. Effects of memory in pseudoproxies on detection and attribution}

We perform detection and attribution studies for the period of 1300-1710 in order to evaluate if the previously observed low amplitude of fingerprints in proxies might be due to memory effects. We chose the upper end of this period to exclude an overlap with the fitting period (1710-1988) and the lower end to ensure reasonable data quality and coverage. Additional sensitivity tests were performed for the slightly longer period of 1300-1850. The proxy reconstructions served as the regression targets, while the fingerprints of external forcing were PPE versions of the all forcings and volcanic forcing only simulations (Fig. 8). Neither the proxy reconstruction nor fingerprints were smoothed prior to the regression. The fingerprints are most affected for the RW version of volcanic forcing only, where the temperature anomalies deviate strongly from the PPE NoM reference at certain periods.

All target datasets show increased volcanic scaling factors for PPE AR3 and PPE LTM compared to PPE NoM (Figs. 9a-c). The addition of memory to the fingerprints makes the model consistent with the proxy 


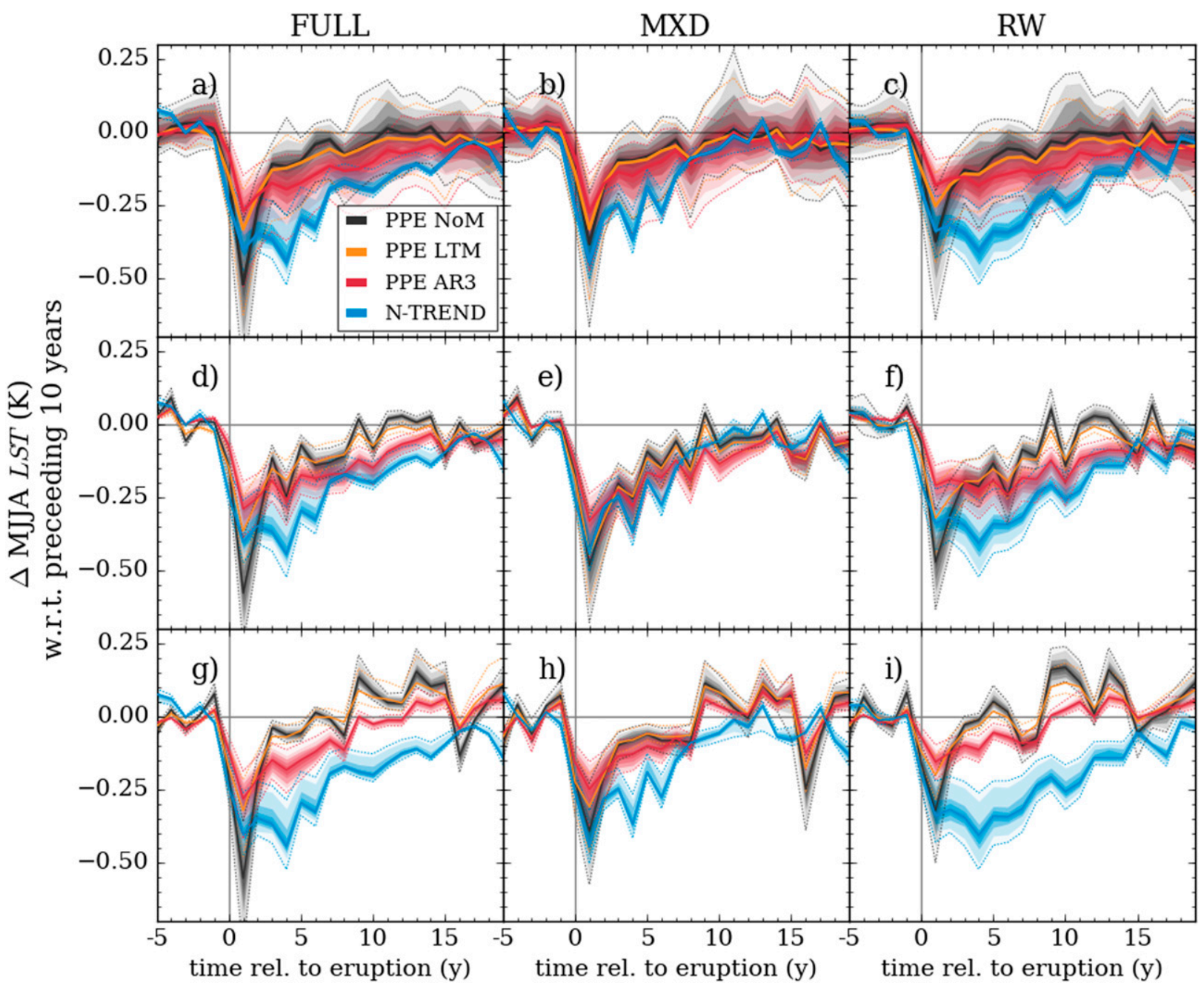

FIG. 7. Superposed epoch analysis for 16 well-dated volcanic eruptions between 1000 and 1900. Year 0 refers to the year of eruption. (a)-(c) Full ensemble range. Shading is as in previous figures. (d)-(f) Best matching ensemble member including reconstruction uncertainty (shaded). (g)-(i) Poorly matching ensemble member.

data in case of the longer period. The highest difference between the memory PPEs and PPE NoM can be observed in the RW reconstruction. For this dataset the scaling factors for volcanic forcing are increased up to the median value $\beta=1.5$. The scaling factors also increase with memory for FULL and MXD; however, the difference to the reference PPE NoM is smaller. These observations are consistent with the results of the epoch analysis, which showed that anomalies in response to volcanic forcing are reduced. Two main observations can be made from plotting the scaled fingerprints relative to their proxy targets (Figs. 9d-f), which are clearly present in FULL and RW, but only weakly present in MXD. The big drop of NH temperature following eruptions in the mid-fifteenth century is matched much better by the memory PPEs in both magnitude and length. The same applies to the eruptions in 1600 and
1640. Low-frequency variability increases for the memory fingerprints, resulting in a better fit for RW and FULL reconstructions, which show a substantial low-frequency variability between 1450 and 1600 . When targeting the period 1300-1850 (Fig. 10) the scaling factors are slightly reduced and in all cases are consistent with one. This could be explained by overfitting the peak warmth in the sixteenth century in the shorter analysis (cf. Figs. 9 and 10). Note that the longer period is also influenced by the wrong dating of Laki (1761 instead of 1783) in the CESM-LME, which could influence the results and dampen the scaling factors.

The residual variability in reconstructions not explained by the fingerprints (Figs. 11a-c) shows a slight decrease when accounting for memory, which is particularly prominent in the RW case. Even though the proxy uncertainty is relatively high, the ensemble 


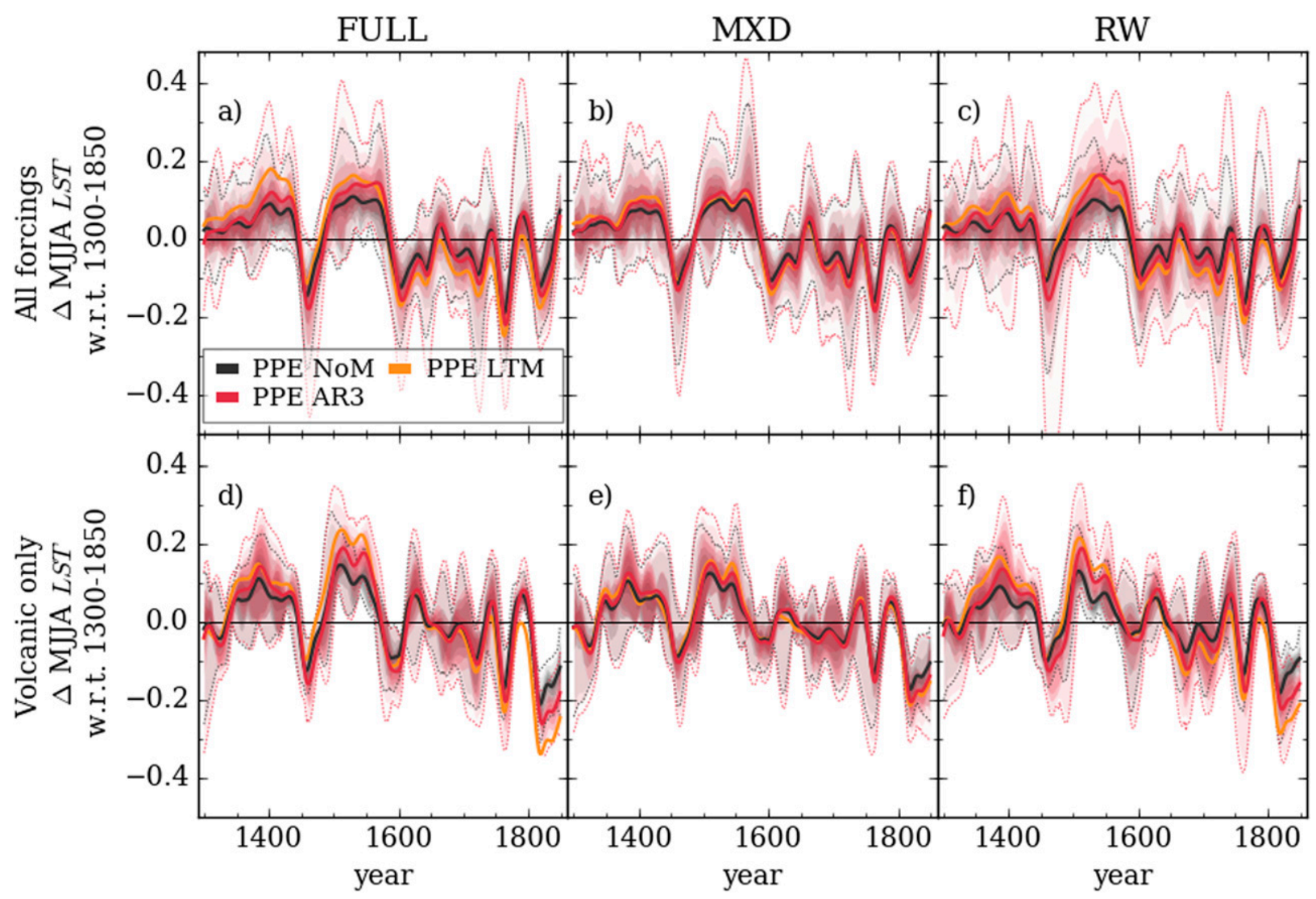

FIG. 8. Pseudoproxy fingerprints of external forcings for the PPE ensembles targeting the (a),(d) full, (b),(e) MXD, and (c),(f) RW-only network. Red and black shading indicates the percentiles of the PPE AR3 and PPE NoM ensembles, respectively. Fingerprints are smoothed using a 20-yr low-pass filter for visualization purposes.

median shows a clear decrease when accounting for memory. Simultaneously, the variance of the PPE control runs decreases and approaches the proxy value. Thus, the residual variability becomes consistent with the control variability for PPE AR3 and higher memory in case of FULL and RW, while for MXD it is consistent for all memory PPEs.

We conclude that models and proxy reconstructions are consistent when accounting for memory effects in $\mathrm{RW}$ data. This indicates better correspondence between signal amplitudes in fingerprints and reconstructions.

\section{Discussion and conclusions}

The implementation of memory improved the agreement between proxy and pseudoproxy reconstructions. Ring-width-only reconstructions have particularly benefited, but results for the full network reconstruction including both width and density proxies were also improved. Although it has long been well known that ring width data can be successfully fitted by an autoregressive memory model (Cook et al. 2002; Meko 1997), we find, for the first time, that implementing autoregressive memory in climate model data can introduce almost identical spectral behavior in model data and resolve proxy-model discrepancies such as the low signal amplitude of the volcanic signal in detection and attribution studies. An autoregressive process of third order performs best out of all our memory models considered. The remarkable agreement between the spectral density of RW only proxy reconstruction and PPE AR3 suggests that even though RW has a clear spectral bias, it is sensitive to the full range of the climate signal. A similarly good agreement was found for the full network, in particular for multidecadal time scales, when the ensemble mean agrees well with PPE AR3. As a consequence of memory biases low-frequency variability is inflated while high-frequency variability is suppressed. This could lead to an overestimation of the magnitude of long-term anomalies, especially for RW data. This phenomenon is robust for all three datasets, where it leads to a warmer MCA, a cooler LIA, and increased warming during the twentieth century in the PPEs when including memory. The effect on the 

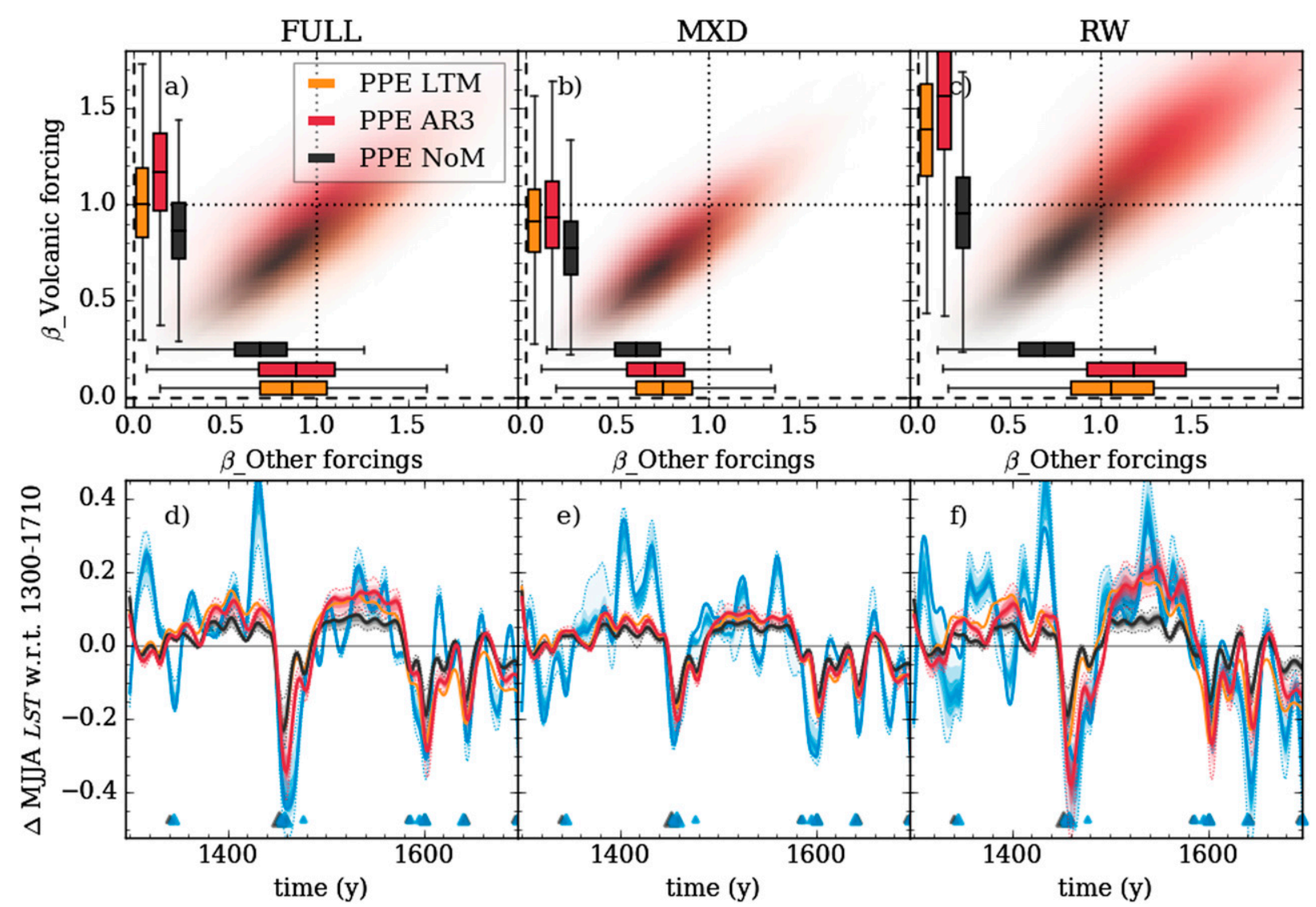

FIG. 9. Results for detection and attribution analysis for the period 1300-1710. (a)-(c) Scaling factors indicating the magnitude of the fingerprints in reconstructions. Box: lower and upper quartiles; line: median; whiskers: 5th-95th percentiles. (d)-(f) Scaled PPE fingerprints against targeted proxy reconstruction (blue) during the regression period smoothed with a 15-yr low-pass filter.

amplitude of the MCA is particularly high, which could be caused by poor data coverage further exacerbating the bias. Without considering memory, MXD reconstructions are most consistent with model simulations. MXD data show little autocorrelation and long-term memory compared to RW and improvements when fitting memory to the PPEs are small. However, reconstructions using density only still show more autocorrelation and long-term memory than observations and model simulations. It remains unclear from our results if the deviations between MXD and observations/ simulations arise from biases in the signal of density proxies or in the simulation of persistence of climate signal in the CESM.

The year-to-year memory causes a dampened amplitude in response to volcanic forcing along with a slower recovery, particularly affecting ring width reconstructions. This confirms earlier studies (Esper et al. 2015; Franke et al. 2013; Schneider et al. 2015; Stoffel et al. 2015). Our results from the epoch analysis tie in with Neukom et al. (2018), who found that the addition of first-order autoregressive $[\mathrm{AR}(1)]$ noise in pseudoproxy reconstructions would slightly dampen the amplitude, but not cause a prolonged cooling. We have, for the first time, provided a memory model that can explain the dampening and the prolonged cooling in proxy reconstructions and resolve the divergence between proxy and climate model response. We have shown that autoregressive memory processes cause a significant reduction of posteruption temperatures for several years. A particular mismatch between PPEs and proxy targets is present in all datasets after around 5 years. This could be explained by internal variability or potentially a lack of short-term feedbacks in the climate model and can be resolved by PPE AR3 for specific ensemble members.

Our results from detection and attribution studies indicate that model simulations and proxy reconstructions agree better when accounting for biological-based memory. While the scaling factors are increased, the residuals are reduced to an extent that is consistent with the model implementation of internal variability. Residuals are smallest for the full network, which is likely a result of higher data coverage, including more 

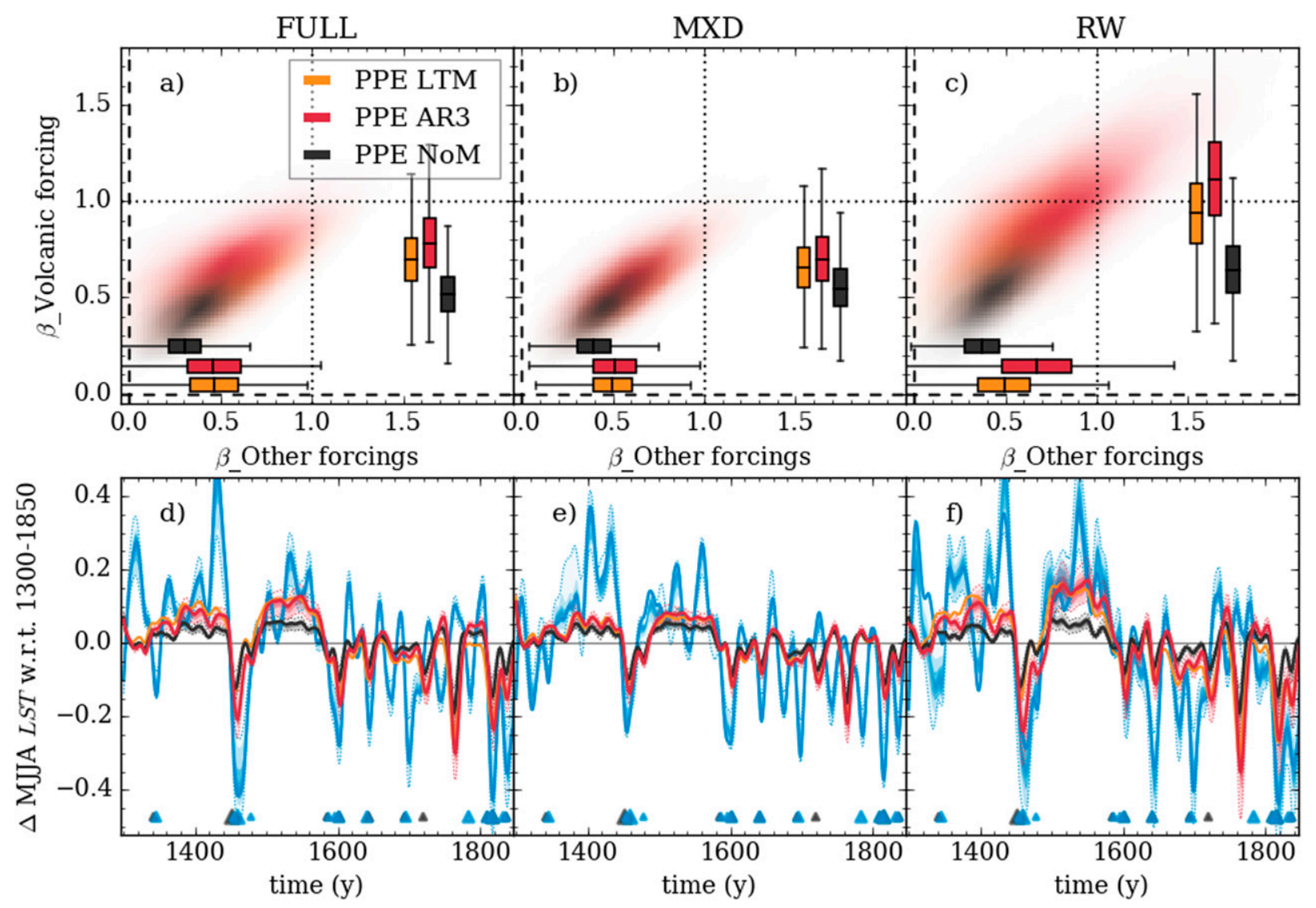

FIG. 10. As Fig. 9, but for the period 1300-1850.

than twice the amount of proxy records as MXD-/RWonly reconstructions. Our results indicate that for both periods the influence of internal variability is low compared to forced variability. When the fingerprints account for memory effects, more forced variability can be detected in the proxy reconstructions, this concerns particularly the variability related to volcanic forcing. The magnitude of the resulting scaling factors varies across the target datasets, with smallest values in case of MXD and highest values in case of RW. This observation holds for both analyzed periods. For the period 1300-1710 the scaling factor for volcanic forcing obtained from the RW target dataset is significantly higher than one, and the low-frequency variability trend during the sixteenth century is extremely well fitted by the scaled PPE AR3 fingerprints. This indicates a potential overfit and does not occur when extending the analysis to 1850 . However, the longer period includes wrongly dated volcanos in the model and thus results are not fully reliable. The persistence of the climate signal due to biological memory processes introduces a degree of smoothing to the proxy reconstructions. This could explain previous observations that using smoothed fingerprints for detection and attribution studies results in higher scaling factors than using unsmoothed fingerprints (Schurer et al. 2013, 2014).

We conclude that it would be beneficial to include ring width into proxy reconstructions, as they agree well with the climate model signal. However, spectral biases have to be considered when comparing model and proxy data. While we have been focusing on treering data in this analysis, it is likely that memory biases of this kind will similarly affect other biological proxy archives, and thus propagate into multiproxy studies. It is beyond the scope of this article to analyze the exact implications on calibration of proxy data. However, our results suggest that it is beneficial for the quality of RW data to invert autoregressive models to extract the real underlying climate signal. Given the sensitivity of low-frequency variability to statistical processing, we conclude that the MCA-LIA difference is not a robust measure for model performance. When comparing model and proxies, spectral biases should be taken into account. Particularly for TLS-like calculations, where model and proxy reconstructions are 


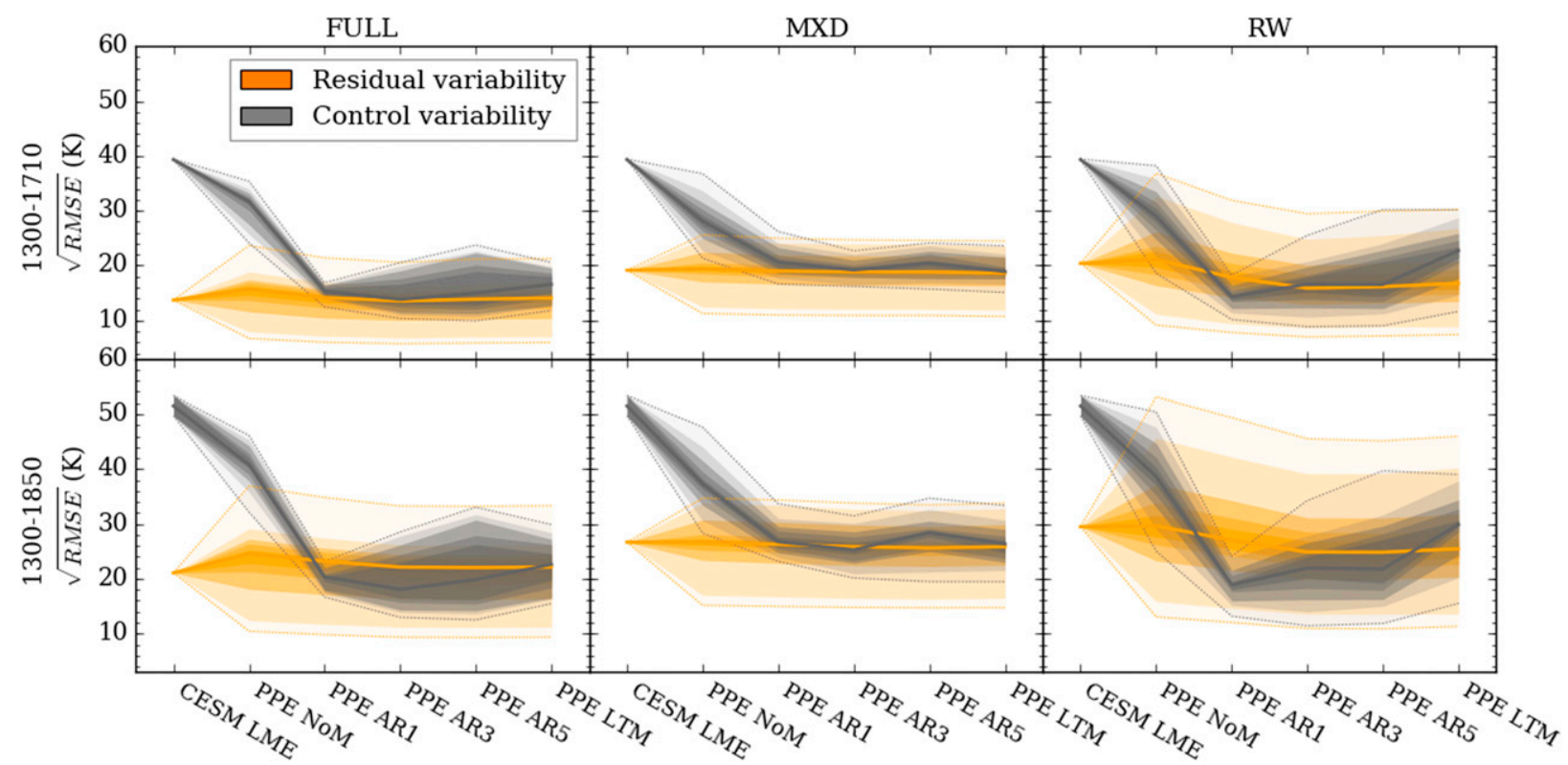

FIG. 11. Unexplained residual variability from the detection and attribution analysis (orange) and square root of sum of squares of equivalent time slice of control variability shown in PPE versions of the CESM LME control simulation (gray).

assumed to have a similar noise structure, it would be beneficial to take into account that certain types of proxy data might not capture high-frequency variability and are subject to inflated low-frequency variability.

Acknowledgments. L.L. was supported by a studentship from the Natural Environment Research Council (NERC) E3 Doctoral training partnership (Grant NE/ L002558/1). A.S. and G.H. were supported by NERC under the Belmont forum, Grant PacMedy (NE/ P006752/1). G.H. was further funded by the Wolfson Foundation and the Royal Society as a Royal Society Wolfson Research Merit Award (WM130060) holder. We acknowledge the National Center for Atmospheric Research (NCAR) for producing and making publicly available their model output. We acknowledge the Northern Hemisphere Tree-Ring Network Development (N-TREND) for providing publicly available data. The authors declare no conflicts of interest. The datasets and code generated and/or analyzed during the current study are available from the corresponding author on request.

\section{REFERENCES}

Allen, M. R., and S. F. B. Tett, 1999: Checking for model consistency in optimal fingerprinting. Climate Dyn., 15, 419-434, https://doi.org/10.1007/s003820050291.

Anchukaitis, K., and Coauthors, 2012: Tree rings and volcanic cooling. Nat. Geosci., 5, 836-837, https://doi.org/10.1038/ ngeo1645.
—-, and Coauthors, 2017: Last millennium Northern Hemisphere summer temperatures from tree rings: Part II, spatially resolved reconstructions. Quat. Sci. Rev., 163, 1-22, https:// doi.org/10.1016/j.quascirev.2017.02.020.

Björklund, J. A., B. E. Gunnarson, K. Seftigen, J. Esper, and H. W. Linderholm, 2014: Blue intensity and density from northern Fennoscandian tree rings, exploring the potential to improve summer temperature reconstructions with earlywood information. Climate Past, 10, 877-885, https://doi.org/10.5194/ cp-10-877-2014.

Böhm, R., P. D. Jones, J. Hiebl, D. Frank, M. Brunetti, and M. Maugeri, 2010: The early instrumental warm-bias: A solution for long central European temperature series 17602007. Climatic Change, 101, 41-67, https://doi.org/10.1007/ s10584-009-9649-4.

Box, G. E. P., 2016: Time Series Analysis: Forecasting and Control. 5th ed. John Wiley \& Sons, 712 pp.

Braconnot, P., S. P. Harrison, M. Kageyama, P. J. Bartlein, V. Masson-Delmotte, A. Abe-Ouchi, B. Otto-Bliesner, and Y. Zhao, 2012: Evaluation of climate models using palaeoclimatic data. Nat. Climate Change, 2, 417-424, https://doi.org/ 10.1038/nclimate1456.

Brohan, P., J. J. Kennedy, I. Harris, S. F. B. Tett, and P. D. Jones, 2006: Uncertainty estimates in regional and global observed temperature changes: A new data set from 1850. J. Geophys. Res., 111, D12106, https://doi.org/10.1029/2005JD006548.

Bryce, R. M., and K. B. Sprague, 2012: Revisiting detrended fluctuation analysis. Sci. Rep., 2, 315, https://doi.org/10.1038/ srep00315.

Bürger, G., I. Fast, and U. Cubasch, 2006: Climate reconstruction

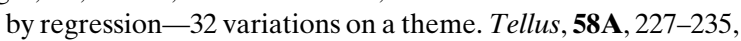
https://doi.org/10.1111/j.1600-0870.2006.00164.x.

Campbell, R., D. McCarroll, N. J. Loader, H. Grudd, I. Robertson, and R. Jalkanen, 2007: Blue intensity in Pinus sylvestris treerings: Developing a new palaeoclimate proxy. Holocene, 17, 821-828, https://doi.org/10.1177/0959683607080523. 
Christiansen, B., T. Schmith, and P. Thejll, 2009: A surrogate ensemble study of climate reconstruction methods: Stochasticity and robustness. J. Climate, 22, 951-976, https://doi.org/ 10.1175/2008JCLI2301.1.

Cook, E. R., and N. Pederson, 2010: Uncertainty, emergence, and statistics in dendrochronology. Dendroclimatology, Springer, 77-112, https://doi.org/10.1007/978-1-4020-5725-0_4.

— R. D. D'Arrigo, and M. E. Mann, 2002: A well-verified, multiproxy reconstruction of the winter North Atlantic oscillation index since A.D. 1400. J. Climate, 15, 1754-1764, https://doi.org/ 10.1175/1520-0442(2002)015<1754:AWVMRO > 2.0.CO;2.

D'Arrigo, R., R. Wilson, and G. Jacoby, 2006: On the long-term context for late twentieth century warming. J. Geophys. Res., 111, D03103, https://doi.org/10.1029/2005JD006352.

, B. Liepert, and P. Cherubini, 2008: On the 'divergence problem' in northern forests: A review of the tree-ring evidence and possible causes. Global Planet. Change, 60, 289305, https://doi.org/10.1016/j.gloplacha.2007.03.004.

$\longrightarrow,-$, and K. J. Anchukaitis, 2013: Volcanic cooling signal in tree ring temperature records for the past millennium. J. Geophys. Res. Atmos., 118, 9000-9010, https://doi.org/10.1002/ JGRD.50692.

Driscoll, S., A. Bozzo, L. J. Gray, A. Robock, and G. Stenchikov, 2012: Coupled Model Intercomparison Project 5 (CMIP5) simulations of climate following volcanic eruptions. J. Geophys. Res., 117, D17105, https://doi.org/10.1029/2012JD017607.

Esper, J., D. C. Frank, and R. J. S. Wilson, 2004: Climate reconstructions: Low-frequency ambition and high-frequency ratification. Eos, Trans. Amer. Geophys. Union, 85, 113-120, https://doi.org/10.1029/2004EO120002.

—, R. J. Wilson, D. C. Frank, A. Moberg, H. Wanner, and J. Luterbacher, 2005: Climate: Past ranges and future changes. Quat. Sci. Rev., 24, 2164-2166, https://doi.org/10.1016/ j.quascirev.2005.07.001.

— L. Schneider, J. E. Smerdon, B. R. Schöne, and U. Büntgen, 2015: Signals and memory in tree-ring width and density data. Dendrochronologia, 35, 62-70, https://doi.org/10.1016/ j.dendro.2015.07.001.

Frank, D., U. Büntgen, R. Böhm, M. Maugeri, and J. Esper, 2007: Warmer early instrumental measurements versus colder reconstructed temperatures: Shooting at a moving target. Quat. Sci. Rev., 26, 3298-3310, https://doi.org/10.1016/ j.quascirev.2007.08.002.

_ J. Esper, E. Zorita, and R. Wilson, 2010a: A noodle, hockey stick, and spaghetti plate: A perspective on high-resolution paleoclimatology. Wiley Interdiscip. Rev.: Climate Change, $\mathbf{1}$, 507-516, https://doi.org/10.1002/wcc.53.

,-- C. C. Raible, U. Büntgen, V. Trouet, B. Stocker, and F. Joos, 2010b: Ensemble reconstruction constraints on the global carbon cycle sensitivity to climate. Nature, 463, 527530, https://doi.org/10.1038/nature08769.

Franke, J., D. Frank, C. C. Raible, J. Esper, and S. Brönnimann, 2013: Spectral biases in tree-ring climate proxies. Nat. Climate Change, 3, 360-364, https://doi.org/10.1038/nclimate1816.

Fredriksen, H.-B., and K. Rypdal, 2016: Spectral characteristics of instrumental and climate model surface temperatures. J. Climate, 29, 1253-1268, https://doi.org/10.1175/ JCLI-D-15-0457.1.

Fritts, H. C., 1976: Tree Rings and Climate. Academic Press, 582 pp.

Gao, C., A. Robock, and C. Ammann, 2008: Volcanic forcing of climate over the past 1500 years: An improved ice core-based index for climate models. J. Geophys. Res., 113, D23111, https://doi.org/10.1029/2008JD010239.
Hegerl, G., and F. Zwiers, 2011: Use of models in detection and attribution of climate change. Wiley Interdiscip. Rev.: Climate Change, 2, 570-591, https://doi.org/10.1002/wcc.121.

—, T. J. Crowley, S. K. Baum, K.-Y. Kim, and W. T. Hyde, 2003: Detection of volcanic, solar and greenhouse gas signals in paleo-reconstructions of Northern Hemispheric temperature. Geophys. Res. Lett., 30, 1242, https://doi.org/10.1029/ 2002 GL016635.

Hegerl, G. C., T. J. Crowley, M. Allen, W. T. Hyde, H. N. Pollack, J. Smerdon, and E. Zorita, 2007: Detection of human influence on a new, validated 1500-year temperature reconstruction. J. Climate, 20, 650-666, https://doi.org/10.1175/JCLI4011.1.

Helama, S., N. G. Makarenko, L. M. Karimova, O. A. Kruglun, M. Timonen, J. Holopainen, J. Meriláinen, and M. Eronen, 2009: Dendroclimatic transfer functions revisited: Little Ice Age and Medieval Warm Period summer temperatures reconstructed using artificial neural networks and linear algorithms. Ann. Geophys., 27, 1097-1111, https://doi.org/10.5194/ angeo-27-1097-2009.

Jones, P., and Coauthors, 2009: High-resolution palaeoclimatology of the last millennium: A review of current status and future prospects. Holocene, 19, 3-49, https://doi.org/10.1177/ 0959683608098952.

Krakauer, N. Y., and J. T. Randerson, 2003: Do volcanic eruptions enhance or diminish net primary production? Evidence from tree rings. Global Biogeochem. Cycles, 17, 1118, https://doi.org/ 10.1029/2003GB002076.

Landrum, L., B. L. Otto-Bliesner, E. R. Wahl, A. Conley, P. J. Lawrence, N. Rosenbloom, and H. Teng, 2013: Last millennium climate and its variability in CCSM4. J. Climate, 26, 1085-1111, https://doi.org/10.1175/JCLI-D-11-00326.1.

Lee, T. C., F. W. Zwiers, and M. Tsao, 2008: Evaluation of proxybased millennial reconstruction methods. Climate Dyn., 31, 263-281, https://doi.org/10.1007/s00382-007-0351-9.

Lough, J. M., and H. C. Fritts, 1987: An assessment of the possible effects of volcanic eruptions on North American climate using tree-ring data, 1602 to 1900 A.D. Climatic Change, 10, 219239, https://doi.org/10.1007/BF00143903.

Mass, C. F., and D. A. Portman, 1989: Major volcanic eruptions and climate: A critical evaluation. J. Climate, 2, 566-593, https:// doi.org/10.1175/1520-0442(1989)002<0566:MVEACA >2.0.CO;2.

Masson-Delmotte, V., and Coauthors, 2013: Information from paleoclimate archives. Climate Change 2013: The Physical Science Basis, T. F. Stocker et al., Eds., Cambridge University Press, 383-464.

Matalas, N. C., 1962: Statistical properties of tree ring data. Int. Assoc. Sci. Hydrol. Bull., 7, 39-47, https://doi.org/10.1080/ 02626666209493254.

Meko, D., 1997: Dendroclimatic reconstruction with time varying predictor subsets of tree indices. J. Climate, 10, 687-696, https://doi.org/10.1175/1520-0442(1997)010<0687: DRWTVP $>2.0 . \mathrm{CO} ; 2$.

Neukom, R., and Coauthors, 2014: Inter-hemispheric temperature variability over the past millennium. Nat. Climate Change, 4, 362-367, https://doi.org/10.1038/nclimate2174.

— A. P. Schurer, N. J. Steiger, and G. C. Hegerl, 2018: Possible causes of data model discrepancy in the temperature history of the last millennium. Sci. Rep., 8, 7572, https://doi.org/10.1038/ s41598-018-25862-2.

- and Coauthors, 2019: Consistent multidecadal variability in global temperature reconstructions and simulations over the Common Era. Nat. Geosci., 12, 643-649, https://doi.org/ 10.1038/S41561-019-0400-0. 
Osborn, T., 2013: Crutem4.2.0.0-2013-03: Climatic Research Unit (CRU) gridded dataset of global historical near-surface air temperature anomalies over land (version 4.2.0.0 Jan. 1850 Mar.2013). NERC British Atmospheric Data Centre, accessed 22 May 2015, https://doi.org/10.5285/eecba94f-62f9-4b7c88d3-482f2c93c468.

— construction of climate from tree-ring chronologies. Dendrochronologia , 18, 9-25.

Otto-Bliesner, B. L., and Coauthors, 2016: Climate variability and change since $850 \mathrm{CE}$ : An ensemble approach with the Community Earth System Model. Bull. Amer. Meteor. Soc., 97, 735-754, https://doi.org/10.1175/BAMS-D-14-00233.1.

Parker, D. E., 1994: Effects of changing exposure of thermometers at land stations. Int. J. Climatol., 14, 1-31, https://doi.org/ 10.1002/joc.3370140102.

Peng, C.-K., S. V. Buldyrev, S. Havlin, M. Simons, H. E. Stanley, and A. L. Goldberger, 1994: Mosaic organization of DNA nucleotides. Phys. Rev., 49E, 1685-1689, https://doi.org/10.1103/ PhysRevE.49.1685.

Rydval, M., L.-Å. Larsson, L. McGlynn, B. E. Gunnarson, N. J. Loader, G. H. Young, and R. Wilson, 2014: Blue intensity for dendroclimatology: Should we have the blues? Experiments from Scotland. Dendrochronologia, 32, 191-204, https:// doi.org/10.1016/j.dendro.2014.04.003.

Rypdal, M., and K. Rypdal, 2014: Long-memory effects in linear response models of Earth's temperature and implications for future global warming. J. Climate, 27, 5240-5258, https:// doi.org/10.1175/JCLI-D-13-00296.1.

Schmidt, G. A., and Coauthors, 2011: Climate forcing reconstructions for use in PMIP simulations of the last millennium (v1.0). Geosci. Model Dev., 4, 33-45, https://doi.org/10.5194/gmd-4-33-2011.

__ , and Coauthors, 2012: Climate forcing reconstructions for use in PMIP simulations of the last millennium (v1.1) Geosci. Model Dev., 5, 185-191, https://doi.org/10.5194/ gmd-5-185-2012.

Schneider, L., J. E. Smerdon, U. Büntgen, R. J. S. Wilson, V. S. Myglan, A. V. Kirdyanov, and J. Esper, 2015: Revising midlatitude summer temperatures back to A.D. 600 based on a wood density network. Geophys. Res. Lett., 42, 4556-4562, https://doi.org/10.1002/2015GL063956.

Schulman, E., 1956: Dendroclimatic Changes in Semiarid America University of Arizona Press, $142 \mathrm{pp}$.

Schurer, A. P., G. C. Hegerl, M. E. Mann, S. F. B. Tett, and S. J Phipps, 2013: Separating forced from chaotic climate variability over the past millennium. J. Climate, 26, 6954-6973, https://doi.org/10.1175/JCLI-D-12-00826.1.

_ S. F. B. Tett, and G. C. Hegerl, 2014: Small influence of solar variability on climate over the past millennium. Nat. Geosci. 7, 104-108, https://doi.org/10.1038/ngeo2040.

Smerdon, J. E., 2012: Climate models as a test bed for climate reconstruction methods: Pseudoproxy experiments. Wiley Interdiscip. Rev.: Climate Change, 3, 63-77, https://doi.org/ 10.1002/wcc.149.

Stevenson, S., J. T. Fasullo, B. L. Otto-Bliesner, R. A. Tomas, and C. Gao, 2017: Role of eruption season in reconciling model and proxy responses to tropical volcanism. Proc. Natl. Acad. Sci. USA, 114, 1822-1826, https://doi.org/10.1073/pnas.1612505114.
St. George, S., 2014: An overview of tree-ring width records across the Northern Hemisphere. Quat. Sci. Rev., 95, 132-150, https:// doi.org/10.1016/j.quascirev.2014.04.029.

—, and T. R. Ault, 2014: The imprint of climate within Northern Hemisphere trees. Quat. Sci. Rev., 89, 1-4, https://doi.org/ 10.1016/j.quascirev.2014.01.007.

Stoffel, M., and Coauthors, 2015: Estimates of volcanic-induces cooling in the Northern Hemisphere over the past 1500 years. Nat. Geosci., 8, 784-788, https://doi.org/10.1038/ngeo2526.

Stokes, M. A., and T. L. Smiley, 1968: An Introduction to Tree-Ring Dating. University of Arizona Press, 73 pp.

Stott, P. A., S. F. B. Tett, G. S. Jones, M. R. Allen, W. J. Ingram, and J. F. B. Mitchell, 2001: Attribution of twentieth century temperature change to natural and anthropogenic causes. Climate Dyn., 17, 1-21, https://doi.org/10.1007/PL00007924.

Timmreck, C., 2012: Modeling the climatic effects of large explosive volcanic eruptions. Wiley Interdiscip. Rev.: Climate Change, 3, 545-564, https://doi.org/10.1002/wcc.192.

Toohey, M., and M. Sigl, 2017: Volcanic stratospheric sulfur injections and aerosol optical depth from 500 BCE to 1900 CE. Earth Syst. Sci. Data, 9, 809-831, https://doi.org/10.5194/ essd-9-809-2017.

Vaganov, E. A., K. J. Anchukaitis, and M. N. Evans, 2010: How well understood are the processes that create dendroclimatic records? A mechanistic model of the climatic control on conifer tree-ring growth dynamics. Dendroclimatology, Springer, 37-75, https://doi.org/10.1007/978-1-4020-5725-0_3

Von Storch, H., 2004: Reconstructing past climate from noisy data. Science, 306, 679-682, https://doi.org/10.1126/science.1096109. , and F. W. Zwiers, 2002: Statistical Analysis in Climate Research. Cambridge University Press, 484 pp.

$\longrightarrow$, E. Zorita, and F. González-Rouco, 2009: Assessment of three temperature reconstruction methods in the virtual reality of a climate simulation. Int. J. Earth Sci., 98, 67-82, https://doi.org/ 10.1007/s00531-008-0349-5.

Wilson, R., and Coauthors, 2007: A matter of divergence: Tracking recent warming at hemispheric scales using tree ring data. J. Geophys. Res., 112, D17103, https://doi.org/10.1029/ 2006JD008318.

, and Coauthors, 2016: Last millennium Northern Hemisphere summer temperatures from tree rings: Part I: The long term context. Quat. Sci. Rev., 134, 1-18, https://doi.org/10.1016/ j.quascirev.2015.12.005.

Zanchettin, D., C. Timmreck, O. Bothe, S. J. Lorenz, G. Hegerl, H.-F. Graf, J. Luterbacher, and J. H. Jungclaus, 2013: Delayed winter warming: A robust decadal response to strong tropical volcanic eruptions? Geophys. Res. Lett., 40, 204-209, https:// doi.org/10.1029/2012GL054403.

Zhang, H., N. Yuan, J. Esper, J. Werner, E. Xoplaki, U. Büntgen, K. Treydte, and J. Luterbacher, 2015: Modified climate with long term memory in tree ring proxies. Environ. Res. Lett., 10, 084020, https://doi.org/10.1088/1748-9326/10/8/084020.

Zhang, P., H. W. Linderholm, B. E. Gunnarson, J. Björklund, and D. Chen, 2016: 1200 years of warm-season temperature variability in central Fennoscandia inferred from tree-ring density. Climate Past, 12, 1297-1312, https://doi.org/10.5194/ cp-12-1297-2016. 